Preprint typeset in JHEP style - HYPER VERSION

$\mathrm{ROM} 2 \mathrm{~F} / 2006 / 18$

hep-th/0607224

\title{
String predictions for near future colliders from one-loop scattering amplitudes around D-brane worlds
}

\author{
Massimo Bianchi and Alessio V. Santini \\ Dipartimento di Fisica 8 Sezione I.N.F.N. \\ Università di Roma "Tor Vergata" \\ Via della Ricerca Scientifica, 1 - 00133 Roma ITALY \\ E-mail: Massimo.Bianchi@roma2.infn.it, avs980@katamail.com
}

\begin{abstract}
We derive compact expressions for one-loop scattering amplitudes of four open-string vector bosons around supersymmetric configurations with intersecting or magnetized D-branes on toroidal orbifolds. We check the validity of our formulae against the structure of their singularities and their behaviour under modular transformations to the transverse channel, exposing closed string exchange. We then specialize to the case of forward scattering and compute the total cross section for two massless open string vector bosons on the brane to decay into closed strings in the bulk, relying on the optical theorem. Although not directly related to collider signatures our predictions represent a step forward towards unveiling phenomenological implications of open and unoriented superstrings.
\end{abstract}




\section{Contents}

1. Introduction and conclusions 2

2. Tree level amplitude 3

3. One-loop CP even amplitudes 5

$3.1 \mathcal{N}=4$ sectors: only CP even amplitudes as in $D=10$

$3.2 \mathcal{N}=1,2$ sectors: $\mathrm{CP}$ even amplitudes 8

3.2.1 Two fermion bilinears (6 terms) 9

3.2.2 Three fermion bilinears (4 terms)

3.2 .3 Four fermion bilinears (1 term, 2 structures)

4. Summary of the results for CP even amplitudes 16

4.1 No fermion bilinears 16

4.2 One fermion bilinear

4.3 Two fermion bilinears 16

4.4 Three fermion bilinears 17

4.5 Four fermion bilinears, connected 17

4.6 Four fermion bilinears, disconnected 17

5. CP odd amplitudes in $\mathcal{N}=1$ sectors 17

5.1 Two fermion bilinears (6 terms) 18

5.2 Three fermion bilinears (12 terms) 18

5.3 Four fermion bilinears (21 terms, 3 structures) 19

6. Forward scattering 19

6.1 CP even amplitudes 20

6.2 CP odd amplitudes 21

7. Imaginary part and total cross section 22

8. Comments 26

A. Elliptic functions 28

A.1 Definitions 28

A.2 Pseudo-periodicity and zeroes 29

A.3 Modular Transformations 29

A.4 Useful Identities 30

A.5 Series Expansions 30 


\section{Introduction and conclusions}

Vacuum configurations with open and unoriented strings have proven to be a particularly rich arena where to address compelling phenomenological issues in a string context amenable to explicit computations (see e.g. [1, 2, 3, 3, 4, 5, [6] for comprehensive reviews).

After their systematization [7, 8, 9, 10, 11, 12, 13, 14, 15], including subtle effects such as rank reduction induced by a quantized NS-NS antisymmetric tensor [14, 15, 16, 17] and the minimal coupling of RR p-form potentials in an asymmetric superghost picture [14, 15], these theories have received an enormous boost when their geometric description in terms of D-branes and $\Omega$-planes [18, 19], pioneered in [20, 21], has catalyzed the attention of the community.

Compactifications on toroidal orbifolds [22, 23, 24, 25, 26, 27] with intersecting [28, 29, 30, 31, 32] and/or magnetized [33, 34, 35, 36, 37] branes represent a simple yet interesting class of models where problems connected with the presence of (large) extra dimensions [38, 39, 40], supersymmetry breaking [41, 42, 43, 44, 45] and moduli stabilization [46, 47, 48, 49, 50, 51, 52, 53, 54, 55] can be tackled in a controllable way. The study of interactions that determine the structure of the low energy effective action and higher derivative corrections thereof has lead to enormous effort at tree (disk and sphere) level [56, 57, 58, 59, 60, 61] but relatively little is known at one-loop [62, 63, 64, 65, 66] and beyond [67]. Aim of the present investigation is to improve the situation in view of potential phenomenological application of this kind of analyses in formulating predictions for near future colliders based on models with relatively small string tension and /or large extra dimensions [68, 69, 70, 71].

With this goal in mind, we use the standard NSR formalism 72 to derive compact expressions for one-loop scattering amplitudes of four open-string vector bosons around vacuum configurations with open and unoriented strings preserving some supersymmetry. Remarkably, in addition to the standard elliptic functions, our final formulae only involve two more modular forms denoted by $\mathcal{E}_{\mathcal{N}}$ and $\mathcal{J}_{\mathcal{N}}$ in the following. We check the validity of our results, that generalize and extend the classic results of Green and Schwarz in $D=10$ [73, 74], against the structure of their singularities and the properties under modular transformations to the transverse channel that exposes closed string tree-level exchange.

We then compute the total cross section $\sigma_{\text {tot }}(s)$ for two (massless) open string vector bosons on the brane to decay into closed strings in the bulk around general unoriented vacuum configurations preserving at least $\mathcal{N}=1$ supersymmetry. The optical theorem relates $\sigma_{\text {tot }}(s)$ to the imaginary part of the foward scattering amplitude $\mathcal{A}(s)$. We work to lowest order in $g_{s}$, i.e. $|\operatorname{disk}|^{2} \approx \operatorname{Im}$ (annulus). In principle, one could directly compute the amplitudes for the decay of two massless open string states into (massive) closed string states on the disk. These amplitudes are however plagued with subtle normalization problems and we find it more convenient to extract 
them from the non-planar one-loop forward scattering amplitudes [68, 69, 70, 71].

The relevant contribution to the process corresponds to an amplitude with vector bosons with the same Chan-Paton factors that can annihilate into gauge-singlet closed string states. More general initial states, involving massless open string scalars or fermions can be computed similarly as described in a forthcoming paper [75]. Processes initiated by gauginos, in the Adjoint of the Chan-Paton group, or matter scalars, in chiral multiplets, are not relevant for collider physics. In addition to the presently studied processes initiated by vector bosons, transforming in the Adjoint of the Chan-Paton group and belonging to the untwisted sector of the orbifold with integer modes connecting parallel or equally magnetized branes, one should also consider processes initiated by matter fermions, that may either belong to the untwisted sector or to twisted sectors connecting branes intersecting at angles or with different magnetic fluxes. The latter can be studied in parallel and will be the focus of [75.

Our present analysis exploits remarkable properties of elliptic functions [76] and free field propagators on genus one surfaces with or without boundaries and crosscaps to compute and simplify the worldsheet correlators. After deriving compact expressions for the one-loop non-planar amplitudes under consideration, we specialize them to the case of forward scattering. Extracting their imaginary part, we determine the total cross section for the deacy into closed strings as a function of the remaining Mandelstam variable $s=-\left(p_{1}+p_{2}\right)^{2}=-2 p_{1} \cdot p_{2}$. It shows the expected peak and threshold structure that encodes the properties of the brane configuration in the internal space.

The plan of the paper is as follows. In Section 2 we recall basic formulae for vertex operators and tree level scattering amplitudes. In Section 3 we compute the one-loop contractions relevant for CP even processes. Section 1 contains a summary of the results for the reader who is not interested in the details of the derivations. $\mathrm{CP}$ odd amplitudes receiving contribution only from $\mathcal{N}=1$ sectors are discussed in section 5 . The case of forward scattering both for CP even and CP odd amplitudes is studied in section 6, while the total cross sections for the decay into closed strings in the bulk are discussed in section 7 . We present our final comments and draw perspectives for our future investigation in section 8 .

Some properties of elliptic functions are collected in the appendices that should be consulted for notation and conventions.

\section{Tree level amplitude}

For completeness, comparison and later purposes we report here the results for the tree level (disk) scattering amplitude of four open string gauge bosons. The result is independent of the amount of supersymmetry enjoyed by the vacuum configuration, 
since the relevant vertex operators in the NSR formalism ${ }^{1}$

$$
\begin{aligned}
V_{0}^{a \tilde{a}}(z) & =a^{\mu}\left(\partial X_{\mu}+i p \cdot \psi \psi_{\mu}\right) e^{i p \cdot X}(z) T_{C P}^{a \tilde{a}} \\
V_{-1}^{a \tilde{a}}(z) & =a^{\mu} \psi_{\mu} e^{-\varphi} e^{i p \cdot X}(z) T_{C P}^{a \tilde{a}}
\end{aligned}
$$

with $p^{2}=0, a \cdot p=0$ for BRS invariance and $\tilde{a}=\Omega a$, do not depend on the details of the compactification encoded in the internal CFT, to be specified later on. In other words they only involve the identity operator that has trivial correlators. The matrices $T_{C P}^{a \tilde{a}}$ belong to the adjoint of the Chan-Paton group, i.e. to the $\mathbf{N} \overline{\mathbf{N}}$ for $U(N)$ or to the $\mathbf{N}(\mathbf{N} \pm \mathbf{1}) / \mathbf{2}$ for $S p(N)$ or $S O(N)$.

Vertex operators are inserted on the boundary of the disk that is conformally equivalent to the upper half plane whereby $z_{i}=x_{i} \in \mathbf{R}$. Three $c$ ghost insertions are needed to fix $S L(2, R)$ invariance. The tree level four bosons scattering amplitude is then given by

$$
\begin{aligned}
& \mathcal{A}_{v v \rightarrow v v}^{\text {tree }}\left(p_{i}, a_{i}\right)= \\
& g_{s} \operatorname{tr}\left(T_{1} T_{2} T_{3} T_{4}\right) \int d z_{3}\left\langle c V_{0}\left(z_{1} ; p_{1}, a_{1}\right) c V_{-1}\left(z_{2} ; p_{2}, a_{2}\right) V_{0}\left(z_{3} ; p_{3}, a_{3}\right) c V_{-1}\left(z_{4} ; p_{4}, a_{4}\right)\right\rangle
\end{aligned}
$$

up to permutations of the external legs.

After performing the free field contractions and including the relevant non-cyclic permutations, one finds ${ }^{2}$

$$
\begin{aligned}
& \mathcal{A}_{v v \rightarrow v v}^{\text {tree }}\left(p_{i}, a_{i}\right)=g_{s}(2 \pi)^{4} \delta\left(\sum_{i} p_{i}\right) K_{v v \rightarrow v v}^{\text {tree }}\left(p_{i}, a_{i}\right) \times \\
& \left\{\left[\operatorname{tr}\left(T_{1} T_{2} T_{3} T_{4}\right)+\operatorname{tr}\left(T_{1} T_{4} T_{3} T_{2}\right)\right] B(s, t)+\right. \\
& {\left[\operatorname{tr}\left(T_{1} T_{3} T_{4} T_{2}\right)+\operatorname{tr}\left(T_{1} T_{2} T_{4} T_{3}\right)\right] B(t, u)+} \\
& \left.\left[\operatorname{tr}\left(T_{1} T_{4} T_{2} T_{3}\right)+\operatorname{tr}\left(T_{1} T_{3} T_{2} T_{4}\right)\right] B(u, s)\right\}
\end{aligned}
$$

where

$$
B(s, t)=\int_{0}^{1} d x x^{2 \alpha^{\prime} p_{1} \cdot p_{2}-1}(1-x)^{2 \alpha^{\prime} p_{1} \cdot p_{3}-1}=\frac{\Gamma\left(-\alpha^{\prime} s\right) \Gamma\left(-\alpha^{\prime} t\right)}{\Gamma\left(\alpha^{\prime} u\right)}
$$

is Euler Beta function that appears in the celebrated Veneziano amplitude, $s=$ $-\left(p_{1}+p_{2}\right)^{2}=-2 p_{1} \cdot p_{2}, t=-\left(p_{1}+p_{4}\right)^{2}=-2 p_{1} \cdot p_{4}, u=-\left(p_{1}+p_{3}\right)^{2}=-2 p_{1} \cdot p_{3}$ and thus $s+t+u=0$.

The bosonic kinematic factor $K_{v v \rightarrow v v}^{t r e e}\left(p_{i}, a_{i}\right)$ is totally symmetric (not simply cyclically symmetric!) and reads [73, 74]

$$
\begin{aligned}
& K_{v v \rightarrow v v}^{\text {tree }}\left(p_{i}, a_{i}\right)=-\frac{1}{4}\left(s t a_{1} \cdot a_{3} a_{2} \cdot a_{4}+u s a_{1} \cdot a_{4} a_{2} \cdot a_{3}+t u a_{1} \cdot a_{2} a_{3} \cdot a_{4}\right) \\
& +\frac{s}{2}\left(a_{1} \cdot p_{4} a_{3} \cdot p_{2} a_{2} \cdot a_{4}+a_{2} \cdot p_{3} a_{4} \cdot p_{1} a_{1} \cdot a_{3}+a_{1} \cdot p_{3} a_{4} \cdot p_{2} a_{2} \cdot a_{3}+a_{2} \cdot p_{4} a_{3} \cdot p_{1} a_{1} \cdot a_{4}\right)
\end{aligned}
$$

${ }^{1}$ Unless otherwise stated, we set $\alpha^{\prime}=1 / 2$ henceforth.

${ }^{2}$ We use mostly plus signature for the Lorentz metric $\eta_{\mu \nu}=(-,+,+,+)$. 


$$
\begin{aligned}
& +\frac{t}{2}\left(a_{2} \cdot p_{1} a_{4} \cdot p_{3} a_{3} \cdot a_{1}+a_{3} \cdot p_{4} a_{1} \cdot p_{2} a_{2} \cdot a_{4}+a_{2} \cdot p_{4} a_{1} \cdot p_{3} a_{3} \cdot a_{4}+a_{3} \cdot p_{1} a_{4} \cdot p_{2} a_{2} \cdot a_{1}\right) \\
& +\frac{u}{2}\left(a_{1} \cdot p_{2} a_{4} \cdot p_{3} a_{3} \cdot a_{2}+a_{3} \cdot p_{4} a_{2} \cdot p_{1} a_{1} \cdot a_{4}+a_{1} \cdot p_{4} a_{2} \cdot p_{3} a_{3} \cdot a_{4}+a_{3} \cdot p_{2} a_{4} \cdot p_{1} a_{2} \cdot a_{1}\right)
\end{aligned}
$$

in dimension $D=10$ as well as in lower dimensions. It may be written more compactly in terms of the linearized field strengths

$$
f_{i}^{\mu \nu}=p_{i}^{\mu} a_{i}^{\nu}-p_{i}^{\nu} a_{i}^{\mu}=-f_{i}^{\nu \mu}
$$

thus getting a manifestly gauge invariant expression

$$
\begin{aligned}
& K_{v v \rightarrow v v}^{\text {tree }}\left(a_{i}, p_{i}\right)=\frac{1}{2}\left[\left(f_{1} f_{2} f_{3} f_{4}\right)+\left(f_{1} f_{3} f_{4} f_{2}\right)+\left(f_{1} f_{4} f_{2} f_{3}\right)\right] \\
& -\frac{1}{4}\left[\left(f_{1} f_{2}\right)\left(f_{3} f_{4}\right)+\left(f_{1} f_{3}\right)\left(f_{4} f_{2}\right)+\left(f_{1} f_{4}\right)\left(f_{2} f_{3}\right)\right]
\end{aligned}
$$

where

$$
\left(f_{i} f_{j} f_{k} f_{l}\right)=f_{i \nu}^{\mu} f_{j \rho}^{\nu} f_{k \sigma}^{\rho} f_{l \mu}^{\sigma}
$$

and

$$
\left(f_{i} f_{j}\right)=f_{i \nu}^{\mu} f_{j \mu}^{\nu}
$$

For forward scattering $p_{4}=-p_{1}$ and $p_{3}=-p_{2}$, so that $t=0$ and $u=-s$, $a_{3}=a_{2}$ and $a_{4}=a_{1}$, the kinematic factor drastically simplifies to

$$
K_{v v \rightarrow v v}^{F S, t r e e}=\left(\alpha^{\prime} s\right)^{2} a_{1}^{2} a_{2}^{2}
$$

\section{One-loop CP even amplitudes}

In this section we compute the worldsheet correlators that appear in the one-loop scattering amplitudes (planar, non-planar and non-orientable) of four open string vector bosons for supersymmetric models with intersecting and magnetized branes on orbifolds ${ }^{3}$. For the moment we focus on $\mathrm{CP}$ even amplitudes that recieve contribution from the even spin structure in the NSR formalism. CP odd amplitudes from the odd spin structure are described in the next section.

Up to Chan-Paton factors, the one-loop four vector boson amplitude in the direct channel ('open string' description) reads

$$
\begin{aligned}
& \mathcal{A}_{v v \rightarrow v v}^{1-\text { loop }}\left(p_{i}, a_{i}\right)=g_{s}^{2} \int_{0}^{\infty} \frac{d t}{t} \int_{\mathcal{R}} \prod_{i} d z_{i} \\
& \sum_{\alpha} c_{\alpha}\left\langle V_{0}\left(z_{1} ; p_{1}, a_{1}\right) V_{0}\left(z_{2} ; p_{2}, a_{2}\right) V_{0}\left(z_{3} ; p_{3}, a_{3}\right) V_{0}\left(z_{4} ; p_{4}, a_{4}\right)\right\rangle_{\alpha} .
\end{aligned}
$$

\footnotetext{
${ }^{3}$ Some properties of elliptic functions and propagators are collected in the Appendix that should be consulted for notation and conventions.
} 
The power of the modular parameter $t$ in the denominator takes care of the volume of the conformal Killing group and effectively cancels the integration over the 'center of mass' coordinate the correlator is independent of. Additional negative powers of $t$ will appear as a result of integration over loop momentum. Summation over the spin structures $\alpha$ ( $\alpha=2,3,4$ even, $\alpha=1$ odd) with appropriate coefficients $c_{\alpha}$ implements the GSO projection. Moreover summation over the various kinds of magnetized or intersecting branes, later on labelled by $a=1, \ldots, N_{a}$ with $N_{a}=$ $\operatorname{Tr}_{a}(\mathbf{1})$, and the various sectors of the orbifold, later on labelled by $k=0, \ldots, n-1$ for the case of $\Gamma=\mathbf{Z}_{n}$, is understood.

In the planar case, all the four vectors should belong to the same factor in the Chan-Paton group and the annulus amplitude $\mathcal{A}$ is schematically given by

$$
\mathcal{A}_{v v \rightarrow v v}^{\text {plan }}=\operatorname{tr}_{a}\left(T_{1} T_{2} T_{3} T_{4} W_{k}\right) \operatorname{tr}_{b}\left(W_{k}\right) \mathcal{A}_{a b}(1,2,3,4)
$$

where the discrete Wilson lines $W$ represent the projective embedding of the orbifold group $\Gamma$ in the Chan-Paton group. The open string vertices are inserted on the same boundary of the worldsheet, $z=-\bar{z}$, and the integration region is given by

$$
\mathcal{R}_{\mathcal{A}}^{\text {plan }}=\left\{z_{i}=i y_{i}: 0<y_{1}<y_{2}<y_{3}<y_{4}<I m \tau_{\mathcal{A}}=t / 2\right\}
$$

Non-planar amplitudes receive contribution also when the vector bosons belong to different factors of the Chan-Paton group, i.e. end on different stacks of D-branes. Up to permutations, depending on the choice of the Chan-Paton matrices for the external legs, the corresponding annulus amplitude $\mathcal{A}$ reads

$$
\mathcal{A}_{v v \rightarrow v v}^{\text {nonpl }}=\operatorname{tr}_{a}\left(T_{1} T_{2}\right) \operatorname{tr}_{b}\left(T_{3} T_{4}\right) \mathcal{A}_{a b}(1,2 ; 3,4)
$$

The integration region is given by is

$$
\mathcal{R}_{\mathcal{A}}^{n o n p l}=\left\{z_{1,2}=i y_{1,2}, z_{3,4}=\frac{1}{2}+i y_{3,4}: 0<y_{i}<\operatorname{Im} \tau_{\mathcal{A}}=t / 2\right\}
$$

but otherwise unrestricted. This will play a crucial role later on in section 7 .

For unoriented strings, the only ones where tadpole cancellation can be achieved thanks to the contribution of the $\Omega$-planes, one has to take into account the contribution of the Möbius strip $\mathcal{M}$, too. Up to permutations, it reads

$$
\mathcal{A}_{v v \rightarrow v v}^{u n o r}=\operatorname{tr}_{a}\left(T_{1} T_{2} T_{3} T_{4} W_{2 k}^{\Omega}\right) \mathcal{M}_{a \tilde{a}}(1,2,3,4)
$$

where $W_{2 k}^{\Omega}$ implements the action of the worldsheet parity in the Chan-Paton group. The choice of $N$ 's and $W$ 's as well as of the fluxes and intersection angles is tightly constrained by consistency conditions such as RR-tadpole cancellation [77, 78, 79, 80, 81]. We will assume that such a choice has been made and shall not discuss this issue any further in this paper. Moreover we will not consider phenomena associated to the presence of anomalous $U(1)$ 's discussed in [82, 83, 84, 85]. 
For the Möbius strip $\mathcal{M}$, the integration region is given by

$$
\mathcal{R}_{\mathcal{M}}=\left\{z_{i}=i y_{i}+\frac{1}{2} \delta: 0<y_{1}<y_{2}<y_{3}<y_{4}<\operatorname{Im} \tau_{\mathcal{M}}=t / 2 ; \delta=1,2\right\}
$$

Given the form of the vector emission vertices there are in principle five different kinds of contributions to the worldsheet correlators:

$$
\left\langle(\partial X)^{4}\right\rangle_{\alpha}+" 4 "\left\langle(\partial X)^{3}(\psi \psi)\right\rangle_{\alpha}+" 6 "\left\langle(\partial X)^{2}(\psi \psi)^{2}\right\rangle_{\alpha}+" 4 "\left\langle(\partial X)(\psi \psi)^{3}\right\rangle_{\alpha}+\left\langle(\psi \psi)^{4}\right\rangle_{\alpha} \text {. }
$$

It is easy to check that the first two kinds of terms vanish for any supersymmetric vacuum configuration after summation over the even spin structures or lack of fermionic zero-modes in the odd spin structure. We are thus left with the last three structures.

Contractions of the spacetime bosonic coordinates, satisfying Neumann boundary conditions, are performed by means of

$$
\mathcal{G}_{\Sigma}(z-w)=\frac{1}{2}\left[\mathcal{G}_{\mathcal{T}}(z-w)+\mathcal{G}_{\mathcal{T}}(z-\tilde{w})+\mathcal{G}_{\mathcal{T}}(\tilde{z}-w)+\mathcal{G}_{\mathcal{T}}(\tilde{z}-\tilde{w})\right]
$$

where $\tilde{z}=1-\bar{z}=z$ and $\tilde{w}=1-\bar{w}=w$ for open string insertions on the boundary of $\Sigma=\mathcal{A}, \mathcal{M}$, and $\mathcal{G}_{\mathcal{T}}(z, w)$ is the bosonic propagator (Bargmann kernel) on the covering torus

$$
\mathcal{G}_{\mathcal{T}}(z-w)=-\frac{\alpha^{\prime}}{2}\left[\log \left|\frac{\theta_{1}(z)}{\theta_{1}^{\prime}(0)}\right|^{2}-\frac{2 \pi}{\operatorname{Im} \tau} \operatorname{Im}(z-w)^{2}\right]
$$

with $\tau=\tau_{\Sigma}$.

In the even spin structures, free fermion contractions are performed by means of

$$
\mathcal{S}_{\alpha}(z-w)=\frac{\theta_{\alpha}(z-w)}{\theta_{1}(z-w)} \frac{\theta_{1}^{\prime}(0)}{\theta_{\alpha}(0)}
$$

the fermionic propagator (Szego kernel).

In the odd spin structure, the fermionic propagator may be taken to be

$$
\mathcal{S}_{1}(z-w)=-\partial_{z} \mathcal{G}(z-w)
$$

Contractions are weighted by the partition function

$$
\mathcal{Z}_{\alpha}^{\mathcal{N}}=\langle 1\rangle_{\alpha, k}^{a, b}
$$

whose explicit form, as we will momentarily see depends on the sector under consideration, i.e. on the choice of $k$ and $a, b$ that determine the number of preserved supersymmetries $\mathcal{N}$. 


\section{$3.1 \mathcal{N}=4$ sectors: only $\mathrm{CP}$ even amplitudes as in $D=10$}

Actually for $\mathcal{N}=4$ sectors only the last term in (3.8) contributes, i.e. survives summation over the (even) spin structures. The fermionic contractions give a constant since the lowest derivative, $\alpha^{\prime} \rightarrow 0$ limit, four-vector amplitude is BPS saturated in these sectors.

$\mathcal{N}=4$ open string sectors are characterized by $k=0$ and connect parallel and equally magnetized branes ('neutral' and 'dipole' strings [2, 66]). The partition function is given by

$$
\mathcal{Z}_{\alpha}^{\mathcal{N}=4}=\mathcal{X}_{a b}^{\mathcal{N}}=4 \frac{\theta_{\alpha}^{4}(0)}{\eta^{12}},
$$

where

$$
\mathcal{X}_{a b}^{\mathcal{N}=4}=\frac{\int d^{4} x_{0} \Lambda_{a b}}{2_{G S O} 2_{\Omega} n_{\text {orb }}\left(\alpha^{\prime} t\right)^{2}}
$$

takes care of numerical factors and bosonic zero modes. In particular, $\int d^{4} x_{0}=V_{X}$ is the (regulated) volume of spacetime, $\int d^{4} p_{0} \exp \left(-\pi \alpha^{\prime} p_{0}^{2}\right)=1 /\left(\alpha^{\prime} t\right)^{2}$, and $\Lambda_{a b}$ denotes the 6-dimensional sum over generalized KK momenta. The numerical factors result from the various projections $\mathbf{Z}_{2}^{G S O}, \mathbf{Z}_{2}^{\Omega}$ and $\mathbf{Z}_{n}^{\text {orbifold. }}$.

Up to permutations, the (non)planar annulus amplitude reads

$$
\begin{aligned}
& \mathcal{A}_{a b}(1,2,3,4)\left(p_{i}, a_{i}\right)=g_{s}^{2} \frac{(2 \pi)^{4}}{4 n} \delta\left(\sum_{i} p_{i}\right) K_{v v \rightarrow v v}^{\text {tree }}\left(p_{i} ; a_{i}\right) \times \\
& \int_{0}^{\infty} \frac{d t}{t^{3}} \Lambda_{a b}\left(\tau_{\mathcal{A}}\right) \int_{\mathcal{R}_{\mathcal{A}}^{(n o n) p l a n}} \prod_{k} d z_{k} \prod_{i<j} e^{-p_{i} \cdot p_{j} \mathcal{G}_{\mathcal{A}}\left(z_{i j}\right)}
\end{aligned}
$$

where $\mathcal{G}_{\mathcal{A}}$ is the free bosonic propagator on the boundary of the annulus $\mathcal{A} 3.9$. The integration regions $\mathcal{R}_{\mathcal{A}}^{\text {plan }}$ and $\mathcal{R}_{\mathcal{A}}^{\text {non-plan }}$ have been discussed above. As indicated, the kinematic factor $K_{v v \rightarrow v v}^{t r e e}$ is exactly the same as at tree level.

The unoriented Möbius amplitude reads

$$
\begin{aligned}
& \mathcal{M}_{a \tilde{a}}(1,2,3,4)=g_{s}^{2} \frac{(2 \pi)^{4}}{4 n} \delta\left(\sum_{i} p_{i}\right) K_{v v \rightarrow v v}^{t r e e}\left(p_{i} ; a_{i}\right) \times \\
& \int_{0}^{\infty} \frac{d t}{t^{3}} \Lambda_{a \tilde{a}}\left(\tau_{\mathcal{A}}\right) \int_{\tilde{\mathcal{R}}_{\mathcal{M}}} \prod_{k} d z_{k} \prod_{i<j} e^{-p_{i} \cdot p_{j} \mathcal{G}_{\mathcal{M}}\left(z_{i j}\right)},
\end{aligned}
$$

where $\mathcal{G}_{\mathcal{M}}$ is the free boson propagator on the boundary of the Möbius strip 3.9.

\section{$3.2 \mathcal{N}=1,2$ sectors: $\mathrm{CP}$ even amplitudes}

Let us consider the $\mathcal{N}=1$ and $\mathcal{N}=2$ supersymmetric sectors that can be analyzed in parallel in the even spin structures. The odd spin structure needs a separate analysis. The main difference between the two cases resides in the internal contribution. Our analyses applies to arbitrary choices ('parallel' [2] or 'oblique' [66]) of 
constant abelian magnetic fluxes ${ }^{4}$ or intersecting angles in (supersymmetric) orbifold compactifications.

In the $\mathcal{N}=1$ case one has

$$
\langle 1\rangle_{\alpha, k, \varepsilon}^{a, b}=\mathcal{Z}_{\alpha}^{\mathcal{N}=1}\left(u_{a b}^{I}\right)=\mathcal{X}_{a b}^{\mathcal{N}=1} \frac{\theta_{\alpha}(0)}{\eta^{3}} \prod_{I} \frac{\theta_{\alpha}\left(u_{a b}^{I}\right)}{\theta_{1}\left(u_{a b}^{I}\right)},
$$

where

$$
\mathcal{X}_{a b}^{\mathcal{N}=1}=\frac{\mathcal{I}_{a b} \int d^{4} x_{0}}{2_{G S O} 2_{\Omega} n_{\text {orb }}\left(\alpha^{\prime} t\right)^{2}}
$$

$\mathcal{I}_{a b}$ is some discrete multiplicity, e.g. degeneracy of Landau levels, number of fixed points or intersections,

$$
u_{a b}^{I}=\varepsilon_{a b}^{I} \tau_{\mathcal{A}}+k v_{a b}^{I}
$$

with $\varepsilon_{a b}^{I}$ denoting intersection angles or magnetic shifts and $k v_{a b}^{I}$ implementing some $\mathbf{Z}_{n}$ orbifold projection with $k=0,1, \ldots n-1 . \mathcal{N}=1$ supersymmetry requires $u_{a b}^{I} \neq 0$, with $\sum_{I} u_{a b}^{I}=0(\bmod 1)$.

In the $\mathcal{N}=2$ case one of the $u_{a b}^{I}$ vanishes. For definiteness let us set $u_{a b}^{3}=0$, then $u_{a b}^{1}=-u_{a b}^{2}=u_{a b}(\bmod 1)$ and one obtains

$$
\langle 1\rangle_{\alpha, k, \varepsilon}^{a, b}=\mathcal{Z}_{\alpha}^{\mathcal{N}=2}\left(u_{a b}\right)=\mathcal{X}_{a b}^{\mathcal{N}=2} \frac{\theta_{\alpha}(0)^{2} \theta_{\alpha}\left(u_{a b}\right) \theta_{\alpha}\left(-u_{a b}\right)}{\eta^{6} \theta_{1}\left(u_{a b}\right) \theta_{1}\left(-u_{a b}\right)},
$$

where

$$
\mathcal{X}_{a b}^{\mathcal{N}=2}=\frac{\int d^{4} x_{0} \mathcal{I}_{a b}^{\perp} \Lambda_{a b}^{\|}\left(\tau_{\mathcal{A}}\right)}{2_{G S O} 2_{\Omega} n_{\text {orb }}\left(\alpha^{\prime} t\right)^{2}} .
$$

In addition to the discrete multiplicity $\mathcal{I}_{a b}^{\perp}$ in the twisted or magnetized directions, a sum of generalized KK momenta $\Lambda_{a b}^{\|}\left(\tau_{\mathcal{A}}\right)$ in the untwisted or unmagnetized directions is present.

In both cases the Chan-Paton factors get modified to $\operatorname{tr}_{a}\left(T^{1} \ldots W^{k}\right)$ by the effect of (discrete) Wilson lines $W$ corresponding to the projective embedding of the orbifold group $\Gamma$ in the gauge group. Notice that the unbroken gauge group $G_{a}^{u}$ for branes of type $a$ corresponds to the generators $T_{a}^{u}$ such that $\left[T_{a}^{u}, W_{a}^{k}\right]=0$.

Let us now consider the Wick contractions one at a time.

\subsubsection{Two fermion bilinears (6 terms)}

Up to permutations (six in all) the typical $\left\langle(\partial X)^{2}(\psi \psi)^{2}\right\rangle_{\alpha}$ correlator reads $\left\langle a_{1} \cdot \partial X e^{i p_{1} \cdot X}\left(z_{1}\right) a_{2} \cdot \partial X e^{i p_{2} \cdot X}\left(z_{2}\right) e^{i p_{3} \cdot X}\left(z_{3}\right) e^{i p_{4} \cdot X}\left(z_{4}\right)\right\rangle\left\langle i p_{3} \cdot \psi a_{3} \cdot \psi\left(z_{3}\right) i p_{4} \cdot \psi a_{4} \cdot \psi\left(z_{4}\right)\right\rangle_{\alpha} \cdot$

\footnotetext{
${ }^{4} \mathrm{~A}$ worldsheet description of the non abelian fluxes discussed in 86 is not yet available.
} 
The bosonic correlator yields

$$
\begin{aligned}
& \left\langle a_{1} \cdot \partial X e^{i p_{1} \cdot X}\left(z_{1}\right) a_{2} \cdot \partial X e^{i p_{2} \cdot X}\left(z_{2}\right) e^{i p_{3} \cdot X}\left(z_{3}\right) e^{i p_{4} \cdot X}\left(z_{4}\right)\right\rangle= \\
& {\left[a_{1} \cdot a_{2} \partial_{1} \partial_{2} \mathcal{G}\left(z_{12}\right)-\sum_{i \neq 1} a_{1} \cdot p_{i} \partial_{1} \mathcal{G}\left(z_{1 i}\right) \sum_{j \neq 2} a_{1} \cdot p_{j} \partial_{2} \mathcal{G}\left(z_{2 j}\right)\right] \Pi\left(p_{i}, z_{i}\right),}
\end{aligned}
$$

where $\mathcal{G}$ is the free bosonic propagator defined in (3.9) and

$$
\Pi\left(p_{i}, z_{i}\right)=\prod_{i<j} \exp \left[-p_{i} \cdot p_{j} \mathcal{G}\left(z_{i j}\right)\right] .
$$

The fermionic correlator yields

$$
\left\langle i p_{3} \cdot \psi a_{3} \cdot \psi\left(z_{3}\right) i p_{4} \cdot \psi a_{4} \cdot \psi\left(z_{4}\right)\right\rangle_{\alpha}=-2 \frac{1}{2^{2}}\left(f_{3} f_{4}\right) \mathcal{S}_{\alpha}^{2}\left(z_{34}\right) \mathcal{Z}_{\alpha},
$$

where $\mathcal{S}_{\alpha}$ is the fermionic propagator (Szego kernel) for even spin structures defined in (3.11). Using

$$
\mathcal{S}_{\alpha}^{2}(z-w)=\mathcal{P}(z-w)-e_{\alpha-1}
$$

where $\mathcal{P}(z)$ is Weierstrass $\mathcal{P}$ function

$$
\mathcal{P}(z)=-\partial_{z}^{2} \log \theta_{1}(z)-2 \eta_{1},
$$

with

$$
\eta_{1}=-2 \pi i \partial_{\tau} \log \eta=-\frac{1}{6} \frac{\theta_{1}^{\prime \prime \prime}(0)}{\theta_{1}^{\prime}(0)}
$$

since $\theta_{1}^{\prime}(0)=2 \pi \eta^{3}$, and

$$
e_{\alpha-1}=-4 \pi i \frac{d}{d \tau} \log \frac{\theta_{\alpha}(0 \mid \tau)}{\eta(\tau)}
$$

it is easy to see that only the term $\partial_{\tau} \log \theta_{\alpha}$ in $e_{\alpha-1}$ survives summation over the even spin structures.

For $\mathcal{N}=1$ sectors, one finds

$$
\mathcal{E}_{\mathcal{N}=1}\left(u_{a b}^{I}\right)=\mathcal{X}_{a b}^{\mathcal{N}=1} \sum_{\alpha} \frac{\theta_{\alpha}^{\prime \prime}(0)}{\eta^{3}} \prod_{I} \frac{\theta_{\alpha}\left(u_{a b}^{I}\right)}{\theta_{1}\left(u_{a b}^{I}\right)}=2 \pi \mathcal{X}_{a b}^{\mathcal{N}=1} \sum_{I} \frac{\theta_{1}^{\prime}\left(u_{a b}^{I}\right)}{\theta_{1}\left(u_{a b}^{I}\right)}=2 \pi \mathcal{X}_{a b}^{\mathcal{N}=1} \frac{\mathcal{H}^{\prime}(0)}{\mathcal{H}(0)}
$$

where

$$
\mathcal{H}(z)=\prod_{I} \theta_{1}\left(z+u_{a b}^{I}\right)
$$

and the zero-mode factor $\mathcal{X}_{a b}^{\mathcal{N}=1}$ is defined in (3.19).

For $\mathcal{N}=2$ sectors one finds

$$
\mathcal{E}_{\mathcal{N}=2}\left(u_{a b}\right)=\mathcal{X}_{a b}^{\mathcal{N}=2} \sum_{\alpha} \frac{\theta_{\alpha}^{\prime \prime}(0) \theta_{\alpha}(0) \theta_{\alpha}\left(u_{a b}\right) \theta_{\alpha}\left(-u_{a b}\right)}{\eta^{3} \theta_{1}\left(u_{a b}\right) \theta_{1}\left(-u_{a b}\right)}=4 \pi^{2} \mathcal{X}_{a b}^{\mathcal{N}=2},
$$


where the zero-mode factor $\mathcal{X}_{a b}^{\mathcal{N}=2}$ is defined in (3.22).

Thus, eventually the fermionic correlator

$$
\left\langle i p_{3} \cdot \psi a_{3} \cdot \psi\left(z_{3}\right) i p_{4} \cdot \psi a_{4} \cdot \psi\left(z_{4}\right)\right\rangle_{\text {even }}=-\frac{1}{2}\left(f_{3} f_{4}\right) \mathcal{E}_{\mathcal{N}=1,2}\left(u_{a b}^{I}\right)
$$

turns out to be independent of the insertion points in $\mathcal{N}=1,2$ sectors. As already stated, it receives vanishing contribution from $\mathcal{N}=4$ sectors.

\subsubsection{Three fermion bilinears ( 4 terms)}

Up to permutations (four in all) the correlator $\left\langle(\psi \psi)^{3}(\partial X)\right\rangle_{\alpha}$ can be most conveniently computed by observing that normal ordering of the fermion bilinears : $\psi \psi$ : allows only for a cyclic Lorentz contraction that yields

$$
\left\langle i p_{1} \cdot \psi a_{1} \cdot \psi i p_{2} \cdot \psi a_{2} \cdot \psi i p_{3} \cdot \psi a_{3} \cdot \psi\right\rangle_{\alpha}=-i \frac{2^{3}}{2^{3}}\left(f_{1} f_{2} f_{3}\right) \mathcal{S}_{\alpha}\left(z_{12}\right) \mathcal{S}_{\alpha}\left(z_{23}\right) \mathcal{S}_{\alpha}\left(z_{13}\right) \mathcal{Z}_{\alpha}^{\mathcal{N}}
$$

where $z_{i j}=z_{i}-z_{j}$ and

$$
\left(f_{1} f_{2} f_{3}\right)=f_{1 \nu}^{\mu} f_{2 \rho}^{\nu} f_{3 \mu}^{\rho}
$$

Using the identity

$$
\mathcal{S}_{\alpha}\left(z_{13}\right) \mathcal{S}_{\alpha}\left(z_{23}\right)=\mathcal{S}_{\alpha}\left(z_{12}\right) \omega\left(z_{1}, z_{2}, z_{3}\right)+\mathcal{S}_{\alpha}^{\prime}\left(z_{12}\right)
$$

where

$$
\omega\left(z_{1}, z_{2}, z_{3}\right)=\partial_{1} \log \theta_{1}\left(z_{12}\right)+\partial_{2} \log \theta_{1}\left(z_{23}\right)+\partial_{3} \log \theta_{1}\left(z_{31}\right)
$$

re-combining the two $\mathcal{S}_{\alpha}\left(z_{12}\right)$, using $(\overline{3.27})$ and

$$
\mathcal{S}_{\alpha}(z) \mathcal{S}_{\alpha}^{\prime}(z)=\frac{1}{2} \partial_{z}\left(\mathcal{P}(z)-e_{\alpha-1}\right)=\frac{1}{2} \mathcal{P}^{\prime}(z)
$$

and summing over spin structures yields

$$
\left\langle i p_{1} \cdot \psi a_{1} \cdot \psi i p_{2} \cdot \psi a_{2} \cdot \psi i p_{3} \cdot \psi a_{3} \cdot \psi\right\rangle_{\text {even }}=-i\left(f_{1} f_{2} f_{3}\right) \omega\left(z_{1}, z_{2}, z_{3}\right) \mathcal{E}_{\mathcal{N}}\left(u_{a b}^{I}\right)
$$

which is manifestly symmetric under any permutation of the three insertion points. As already stated, this correlator gets no contribution from $\mathcal{N}=4$ sectors.

The bosonic correlator simply yields

$$
\left\langle e^{i p_{1} \cdot X} e^{i p_{2} \cdot X} e^{i p_{3} \cdot X} a_{4} \cdot \partial X e^{i p_{4} \cdot X}\right\rangle=i \sum_{i \neq 4} a_{4} \cdot p_{i} \partial_{4} \mathcal{G}\left(z_{i 4}\right) \Pi\left(p_{i}, z_{i}\right)
$$

where $\mathcal{G}$ is the bosonic propagator 3.9 and $\Pi\left(p_{i}, z_{i}\right)$ is the momentum factor (3.25). 


\subsubsection{Four fermion bilinears ( 1 term, 2 structures)}

Let us finally consider the $\left\langle(\psi \psi)^{4}\right\rangle_{\alpha}$ term. The bosonic coordinates contribute the momentum factor $\Pi\left(z_{i} ; p_{i}\right)$ defined in (3.25). Taking into account normal ordering of the : $\psi \psi$ :'s allows for two kinds of contraction.

Three are connected contractions of the Lorentz indices that yield

$$
\left\langle(\psi \psi)^{4}\right\rangle_{\alpha}^{c o n n}=\frac{2^{4}}{2^{4}}\left(f_{1} f_{2} f_{3} f_{4}\right) \mathcal{S}_{\alpha}\left(z_{12}\right) \mathcal{S}_{\alpha}\left(z_{23}\right) \mathcal{S}_{\alpha}\left(z_{34}\right) \mathcal{S}_{\alpha}\left(z_{14}\right) \mathcal{Z}_{\alpha}
$$

where

$$
\left(f_{1} f_{2} f_{3} f_{4}\right)=f_{1 \nu}^{\mu} f_{2 \rho}^{\nu} f_{3 \lambda}^{\rho} f_{4 \mu}^{\lambda} .
$$

Using (3.37), the product of fermionic propagators can be simplified to

$$
\begin{aligned}
& {\left[\mathcal{S}_{\alpha}\left(z_{13}\right) \omega\left(z_{1}, z_{2}, z_{3}\right)+\mathcal{S}_{\alpha}^{\prime}\left(z_{13}\right)\right]\left[\mathcal{S}_{\alpha}\left(z_{13}\right) \omega\left(z_{1}, z_{4}, z_{3}\right)+\mathcal{S}_{\alpha}^{\prime}\left(z_{13}\right)\right] } \\
= & \omega\left(z_{1}, z_{2}, z_{3}\right) \omega\left(z_{1}, z_{4}, z_{3}\right)\left[\mathcal{P}\left(z_{13}\right)-e_{\alpha-1}\right] \\
& +\frac{1}{2}\left[\omega\left(z_{1}, z_{2}, z_{3}\right)+\omega\left(z_{1}, z_{4}, z_{3}\right)\right] \mathcal{P}^{\prime}\left(z_{13}\right)+\mathcal{S}_{\alpha}^{\prime}\left(z_{13}\right)^{2} .
\end{aligned}
$$

Summing over spin structures only the first and the last term survive. Let us denote them by $\mathcal{U}_{\mathcal{N}}\left(z_{i}\right)$ and $\mathcal{V}_{\mathcal{N}}\left(z_{i}\right)$. The former simply reads

$$
\mathcal{U}_{\mathcal{N}}\left(z_{i}\right)=-\omega\left(z_{1}, z_{2}, z_{3}\right) \omega\left(z_{1}, z_{4}, z_{3}\right) \mathcal{E}_{\mathcal{N}}\left(u_{a b}^{I}\right) .
$$

The latter is more laborious. Observing that

$$
\mathcal{S}_{\alpha}^{\prime}(z)^{2}=\partial_{z}\left[\mathcal{S}_{\alpha}(z) \mathcal{S}_{\alpha}^{\prime}(z)\right]-\mathcal{S}_{\alpha}(z) \mathcal{S}_{\alpha}^{\prime \prime}(z),
$$

which, using (3.27), in turn gives

$$
\mathcal{S}_{\alpha}^{\prime}(z)^{2}=\frac{1}{2} \mathcal{P}^{\prime \prime}(z)-\mathcal{S}_{\alpha}(z) \mathcal{S}_{\alpha}^{\prime \prime}(z)
$$

it is clear that only the second term contributes after summation over the spin structures, so that

$$
\mathcal{V}_{\mathcal{N}}\left(z_{i}\right)=\lim _{z_{0} \rightarrow z_{1}} \partial_{z_{0}}^{2} \sum_{\alpha} c_{\alpha} \mathcal{S}_{\alpha}\left(z_{03}\right) \mathcal{S}_{\alpha}\left(z_{13}\right) \mathcal{Z}_{\alpha}^{\mathcal{N}} .
$$

Further using (3.37) in the following guise

$$
\mathcal{S}_{\alpha}\left(z_{03}\right) \mathcal{S}_{\alpha}\left(z_{13}\right)=\mathcal{S}_{\alpha}\left(z_{01}\right)\left[\omega_{z_{0}-z_{1}}\left(z_{3}\right)+\frac{\theta_{\alpha}^{\prime}\left(z_{01}\right)}{\theta_{\alpha}\left(z_{01}\right)}\right],
$$

where

$$
\omega_{x-y}(z)=\partial_{z} \log \frac{\theta_{1}(z-x)}{\theta_{1}(z-y)}
$$


is a differential of the third kind with two simple poles with opposite residues $( \pm 1)$ at $z=x$ and $z=y$, yields

$$
\mathcal{V}_{\mathcal{N}}\left(z_{i}\right)=-\lim _{z_{0} \rightarrow z_{1}} \partial_{z_{0}}^{2} \sum_{\alpha} c_{\alpha}\left[\frac{\theta_{\alpha}\left(z_{01}\right)}{\theta_{1}\left(z_{01}\right)} \omega_{z_{0}-z_{1}}\left(z_{3}\right)+\frac{\theta_{\alpha}\left(z_{01}\right)}{\theta_{1}\left(z_{01}\right)}\right] \mathcal{Z}_{\alpha}^{\mathcal{N}}
$$

Both terms can be computed by means of the Riemann identity for even spin structures

$$
\begin{aligned}
& \sum_{\alpha} c_{\alpha} \theta_{\alpha}\left(z_{1}\right) \theta_{\alpha}\left(z_{2}\right) \theta_{\alpha}\left(z_{3}\right) \theta_{\alpha}\left(z_{4}\right)= \\
& \theta_{1}\left(z_{1}^{\prime}\right) \theta_{1}\left(z_{2}^{\prime}\right) \theta_{1}\left(z_{3}^{\prime}\right) \theta_{1}\left(z_{4}^{\prime}\right)-\theta_{1}\left(z_{1}^{\prime \prime}\right) \theta_{1}\left(z_{2}^{\prime \prime}\right) \theta_{1}\left(z_{3}^{\prime \prime}\right) \theta_{1}\left(z_{4}^{\prime \prime}\right),
\end{aligned}
$$

where $z_{i}^{\prime}$ and $z_{i}^{\prime \prime}$ are related to $z_{i}$ through

$$
\begin{array}{rlrl}
z_{1}^{\prime} & =\frac{1}{2}\left(z_{1}+z_{2}+z_{3}+z_{4}\right) & z_{2}^{\prime} & =\frac{1}{2}\left(z_{1}+z_{2}-z_{3}-z_{4}\right) \\
z_{3}^{\prime} & =\frac{1}{2}\left(z_{1}-z_{2}+z_{3}-z_{4}\right) & z_{4}^{\prime}=\frac{1}{2}\left(z_{1}-z_{2}-z_{3}+z_{4}\right)
\end{array}
$$

and

$$
\begin{aligned}
z_{1}^{\prime \prime}=\frac{1}{2}\left(-z_{1}+z_{2}+z_{3}+z_{4}\right) & z_{2}^{\prime \prime}=\frac{1}{2}\left(z_{1}-z_{2}+z_{3}+z_{4}\right) \\
z_{3}^{\prime \prime}=\frac{1}{2}\left(z_{1}+z_{2}-z_{3}+z_{4}\right) & z_{4}^{\prime \prime}=\frac{1}{2}\left(z_{1}+z_{2}+z_{3}-z_{4}\right) .
\end{aligned}
$$

For $\mathcal{N}=1$ sectors

$$
\begin{aligned}
& \mathcal{V}_{\mathcal{N}=1}\left(z_{i}\right)=-2 \pi \mathcal{X}_{a b}^{\mathcal{N}=1} \times \\
& \lim _{z_{0} \rightarrow z_{1}} \partial_{z_{0}}^{2}\left[\frac{\left(\omega_{z_{0}-z_{1}}\left(z_{3}\right)+\partial_{z_{0}}\right)\left\{\theta_{1}\left(z_{01} / 2\right)\left[\mathcal{H}\left(z_{01} / 2\right)-\mathcal{H}\left(-z_{01} / 2\right)\right]\right\}}{\theta_{1}\left(z_{01}\right) \mathcal{H}(0)}\right],
\end{aligned}
$$

where $\mathcal{H}(z)$ is defined in (3.32), that eventually yields

$$
\begin{aligned}
\mathcal{V}_{\mathcal{N}=1}\left(z_{i}\right) & =-2 \pi \mathcal{X}_{a b}^{\mathcal{N}=1} \frac{\mathcal{H}^{\prime}(0)}{\mathcal{H}(0)}\left[\partial_{3} \frac{\theta_{1}^{\prime}\left(z_{31}\right)}{\theta_{1}\left(z_{31}\right)}+\frac{1}{6} \frac{\theta_{1}^{\prime \prime \prime}(0)}{\theta_{1}^{\prime}(0)}-\frac{1}{6} \frac{\mathcal{H}^{\prime \prime \prime}(0)}{\mathcal{H}^{\prime}(0)}\right] \\
& =\mathcal{E}_{\mathcal{N}=1} \mathcal{P}\left(z_{13}\right)+\mathcal{J}_{\mathcal{N}=1},
\end{aligned}
$$

where

$$
\mathcal{J}_{\mathcal{N}=1}=2 \pi \mathcal{X}_{a b}^{\mathcal{N}=1} \frac{\mathcal{H}^{\prime}(0)}{\mathcal{H}(0)}\left[3 \eta_{1}+\frac{1}{6} \frac{\mathcal{H}^{\prime \prime \prime}(0)}{\mathcal{H}^{\prime}(0)}\right] .
$$

Including the bosonic contraction producing the momentum factor (3.25) and adding the term (3.45), one eventually finds

$$
\begin{aligned}
& \left\langle(\psi \psi)^{4}\right\rangle_{\text {conn }}^{\mathcal{N}=1}=\frac{1}{2}\left(f_{1} f_{2} f_{3} f_{4}\right) \Pi\left(p_{i}, z_{i}\right)\left\{2 \mathcal{J}_{\mathcal{N}=1}+\right. \\
& \left.+\mathcal{E}_{\mathcal{N}=1}\left[\mathcal{P}\left(z_{13}\right)-\omega\left(z_{1}, z_{2}, z_{3}\right) \omega\left(z_{1}, z_{4}, z_{3}\right)+\mathcal{P}\left(z_{24}\right)-\omega\left(z_{2}, z_{1}, z_{4}\right) \omega\left(z_{2}, z_{3}, z_{4}\right)\right]\right\}
\end{aligned}
$$


where $\mathcal{E}_{\mathcal{N}=1}$ has been defined above (3.31). Symmetry under the exchange of $z_{1}, z_{3}$ with $z_{2}, z_{4}$ has been made explicit though unnecessary, since we expect it to be

$$
\mathcal{P}\left(z_{31}\right)-\omega\left(z_{1}, z_{2}, z_{3}\right) \omega\left(z_{1}, z_{4}, z_{3}\right)=\mathcal{P}\left(z_{24}\right)-\omega\left(z_{2}, z_{1}, z_{4}\right) \omega\left(z_{2}, z_{3}, z_{4}\right)
$$

from consideration of periodicity and singularity.

For $\mathcal{N}=2$ sectors, ones finds

$$
\mathcal{V}_{\mathcal{N}=2}\left(z_{i}\right)=-4 \pi^{2} \mathcal{X}_{a b}^{\mathcal{N}=2} \lim _{z_{0} \rightarrow z_{1}} \partial_{z_{0}}^{2} \sum_{\alpha} c_{\alpha} \frac{\theta_{\alpha}\left(z_{03}\right)}{\theta_{1}\left(z_{03}\right)} \frac{\theta_{\alpha}\left(z_{13}\right)}{\theta_{1}\left(z_{13}\right)} \frac{\theta_{\alpha}\left(u_{a b}\right)}{\theta_{1}\left(u_{a b}\right)} \frac{\theta_{\alpha}\left(-u_{a b}\right)}{\theta_{1}\left(-u_{a b}\right)}
$$

The limit yields

$$
\begin{aligned}
& \partial_{z_{0}}^{2}\left[\theta_{1}\left(\frac{z_{03}+z_{13}}{2}\right) \theta_{1}\left(\frac{z_{03}+z_{13}}{2}\right) \theta_{1}\left(\frac{z_{03}-z_{13}}{2}-u_{a b}\right) \theta_{1}\left(\frac{z_{03}-z_{13}}{2}+u_{a b}\right)+\right. \\
& \left.\theta_{1}\left(\frac{z_{03}-z_{13}}{2}\right) \theta_{1}\left(\frac{z_{03}-z_{13}}{2}\right) \theta_{1}\left(\frac{z_{03}+z_{13}}{2}-u_{a b}\right) \theta_{1}\left(\frac{z_{03}+z_{13}}{2}+u_{a b}\right)\right]_{z_{0}=z_{1}} \\
& =\left[\mathcal{P}\left(z_{31}\right)-\mathcal{P}\left(u_{a b}\right)\right] \theta_{1}\left(z_{13}\right)^{2} \theta_{1}\left(-u_{a b}\right)^{2} .
\end{aligned}
$$

So that, including the momentum factor (3.25), one eventually obtains

$$
\begin{aligned}
& \left\langle(\psi \psi)^{4}\right\rangle_{\text {conn }}^{\mathcal{N}=2}=\frac{1}{2}\left(f_{1} f_{2} f_{3} f_{4}\right) \Pi\left(p_{i}, z_{i}\right)\left\{2 \mathcal{J}_{\mathcal{N}=2}-\right. \\
& \left.+\mathcal{E}_{\mathcal{N}=2}\left[\mathcal{P}\left(z_{13}\right)-\omega\left(z_{1}, z_{2}, z_{3}\right) \omega\left(z_{1}, z_{4}, z_{3}\right)+\mathcal{P}\left(z_{24}\right)-\omega\left(z_{2}, z_{1}, z_{4}\right) \omega\left(z_{2}, z_{3}, z_{4}\right)\right]\right\}
\end{aligned}
$$

where

$$
\mathcal{J}_{\mathcal{N}=2}=-\mathcal{E}_{\mathcal{N}=2} \mathcal{P}\left(u_{a b}\right)=-4 \pi^{2} \mathcal{X}_{a b}^{\mathcal{N}=2} \mathcal{P}\left(u_{a b}\right)
$$

The other kind of disconnected contractions lead to three inequivalent possibilities that yield terms of the form

$$
\left\langle(\psi \psi)^{4}\right\rangle_{\alpha}^{d i s c}=\frac{2^{2}}{2^{4}}\left(f_{1} f_{2}\right)\left(f_{3} f_{4}\right) \mathcal{S}_{\alpha}^{2}\left(z_{12}\right) \mathcal{S}_{\alpha}^{2}\left(z_{34}\right) \mathcal{Z}_{\alpha}^{\mathcal{N}}
$$

Dropping the kinematical factor, summation over spin structures yields

$$
\left[\mathcal{P}\left(z_{12}\right)+\mathcal{P}\left(z_{34}\right)\right] \mathcal{E}_{\mathcal{N}}+\mathcal{J}_{\mathcal{N}}
$$

where

$$
\mathcal{J}_{\mathcal{N}}=\sum_{\alpha} c_{\alpha} e_{\alpha-1}^{2} \mathcal{Z}_{\alpha}^{\mathcal{N}}
$$

turns out to hold for the $\mathcal{J}_{\mathcal{N}}$ previously defined in (3.57) and (3.63). Indeed, (3.66) can be simplified using (3.37) and 76.

$$
e_{\alpha-1}^{2}=i \pi \partial_{\tau} e_{\alpha-1}+2 \eta_{1} e_{\alpha-1}+\frac{1}{6} g_{2}
$$


to

$$
\mathcal{J}_{\mathcal{N}}=2 \eta_{1} \mathcal{E}_{\mathcal{N}}-\frac{1}{4} \sum_{\alpha} c_{\alpha}\left[\frac{\theta_{\alpha}^{(4)}(0)}{\theta_{\alpha}(0)}-\left(\frac{\theta_{\alpha}^{\prime \prime}(0)}{\theta_{\alpha}(0)}\right)^{2}\right] \mathcal{Z}_{\alpha}^{\mathcal{N}}
$$

In $\mathcal{N}=1$ sectors one finds

$$
\sum_{\alpha} c_{\alpha} \frac{\theta_{\alpha}^{(4)}(0)}{\eta^{3}} \prod_{I} \frac{\theta_{\alpha}\left(u^{I}\right)}{\theta_{1}\left(u^{I}\right)}=\frac{1}{2} \mathcal{E}_{\mathcal{N}=1}\left[\frac{\mathcal{H}^{\prime \prime \prime}}{\mathcal{H}^{\prime}}-6 \eta_{1}\right]
$$

and

$$
\sum_{\alpha} c_{\alpha} \frac{\theta_{\alpha}^{\prime \prime}(0)^{2}}{\eta^{3} \theta_{\alpha}(0)} \prod_{I} \frac{\theta_{\alpha}\left(u^{I}\right)}{\theta_{1}\left(u^{I}\right)}=\frac{1}{6} \sum_{\alpha} c_{\alpha} \frac{\partial_{z}^{4}\left[\theta_{\alpha}(z)^{2}\right]_{z=0}-2 \theta_{\alpha}(0) \theta_{\alpha}^{(4)}(0)}{\eta^{3} \theta_{\alpha}(0)} \prod_{I} \frac{\theta_{\alpha}\left(u^{I}\right)}{\theta_{1}\left(u^{I}\right)}
$$

The second term is given in (3.69), while the first can be computed by means of the identity

$$
\frac{\theta_{\alpha}(z)^{2}}{\theta_{\alpha}(0)}=\frac{\mathcal{S}_{\alpha}(z)^{2} \theta_{1}(z)^{2} \theta_{\alpha}(0)}{\theta_{1}^{\prime}(0)^{2}}=\frac{\left[\mathcal{P}(z)-e_{\alpha-1}\right] \theta_{1}(z)^{2} \theta_{\alpha}(0)}{\theta_{1}^{\prime}(0)^{2}},
$$

that after differentiation and summation over the spin structures yields

$$
\frac{1}{6} 2 \pi \mathcal{X}_{a b}^{\mathcal{N}=1} \sum_{\alpha} c_{\alpha} \frac{\partial_{z}^{4}\left[\theta_{\alpha}(z)^{2}\right]_{z=0}}{\eta^{3} \theta_{\alpha}(0)} \prod_{I} \frac{\theta_{\alpha}\left(u_{a b}^{I}\right)}{\theta_{1}\left(u_{a b}^{I}\right)}=-8 \eta_{1} \mathcal{E}_{\mathcal{N}=1}
$$

so that eventually one indeed finds

$$
\mathcal{J}_{\mathcal{N}=1}=\mathcal{E}_{\mathcal{N}=1}\left[\frac{1}{6} \frac{\mathcal{H}^{\prime \prime \prime}(0)}{\mathcal{H}^{\prime}(0)}+3 \eta_{1}\right]
$$

as above (3.57). Including the momentum factor (3.25) yields

$$
\left\langle(\psi \psi)^{4}\right\rangle_{\text {disc }}^{\mathcal{N}=1}=\frac{1}{4}\left(f_{1} f_{2}\right)\left(f_{3} f_{4}\right)\left\{\mathcal{E}_{\mathcal{N}=1}\left[\mathcal{P}\left(z_{12}\right)+\mathcal{P}\left(z_{34}\right)\right]+\mathcal{J}_{\mathcal{N}=1}\right\} \Pi\left(p_{i}, z_{i}\right)
$$

In $\mathcal{N}=2$ sectors one has

$$
\begin{aligned}
& \mathcal{J}_{\mathcal{N}=2}=-\frac{\mathcal{X}^{\mathcal{N}=2}}{\eta^{6}} \sum_{\alpha} c_{\alpha}\left[\theta_{\alpha}^{\prime \prime}(0)-2 \eta_{1} \theta_{\alpha}(0)\right]^{2} \frac{\theta_{\alpha}\left(u_{a b}\right)^{2}}{\theta_{1}\left(u_{a b}\right)^{2}} \\
& =4 \eta_{1} \mathcal{E}_{\mathcal{N}=2}-\frac{\mathcal{X}_{a b}^{\mathcal{N}=2}}{\eta^{6}} \sum_{\alpha} c_{\alpha} \theta_{\alpha}^{\prime \prime}(0)^{2} \frac{\theta_{\alpha}\left(u_{a b}\right)^{2}}{\theta_{1}\left(u_{a b}\right)^{2}}
\end{aligned}
$$

The last sum leads to

$$
\begin{aligned}
& -\partial_{z}^{2} \partial_{w}^{2}\left[\theta_{1}\left(\frac{z+w}{2}\right) \theta_{1}\left(\frac{z+w}{2}\right) \theta_{1}\left(\frac{z-w}{2}-u_{a b}\right) \theta_{1}\left(\frac{z-w}{2}+u_{a b}\right)+\right. \\
& \left.\theta_{1}\left(\frac{z-w}{2}\right) \theta_{1}\left(\frac{z-w}{2}\right) \theta_{1}\left(\frac{z+w}{2}-u_{a b}\right) \theta_{1}\left(\frac{z+w}{2}+u_{a b}\right)\right]_{z=w=0} \\
& =-\theta_{1}^{\prime}(0)^{2}\left[\partial_{u}^{2} \log \theta_{1}\left(u_{a b}\right)-2 \eta_{1}\right]=\theta_{1}^{\prime}(0)^{2} \mathcal{P}\left(u_{a b}\right) .
\end{aligned}
$$


Including the momentum factor finally yields

$$
\left\langle(\psi \psi)^{4}\right\rangle_{\text {disc }}^{\mathcal{N}=2}=\frac{1}{4}\left(f_{1} f_{2}\right)\left(f_{3} f_{4}\right)\left\{\mathcal{E}_{\mathcal{N}=2}\left[\mathcal{P}\left(z_{12}\right)+\mathcal{P}\left(z_{34}\right)\right]-\mathcal{J}_{\mathcal{N}=2}\right\} \Pi\left(p_{i}, z_{i}\right)
$$

where

$$
\mathcal{J}_{\mathcal{N}=2}=-\mathcal{E}_{\mathcal{N}=2} \mathcal{P}\left(u_{a b}\right)
$$

as above $(3.63)$ and $\mathcal{E}_{\mathcal{N}=2}$ is defined in $(3.33)$.

\section{Summary of the results for CP even amplitudes}

Let us summarize our results in the NSR formalism according to the number of supersymmetries preserved for the $\mathrm{CP}$ even amplitudes receiving contribution from the sum over even spin structures.

\subsection{No fermion bilinears}

$$
\left\langle a_{1} \cdot \partial X e^{i p_{1} \cdot X}\left(z_{1}\right) a_{2} \cdot \partial X e^{i p_{2} \cdot X}\left(z_{2}\right) a_{3} \cdot \partial X e^{i p_{3} \cdot X}\left(z_{3}\right) a_{4} \cdot \partial X e^{i p_{4} \cdot X}\left(z_{4}\right)\right\rangle_{\text {even }}=0
$$

in any supersymmetric sector after summing over the even spin structures.

\subsection{One fermion bilinear}

$$
\left\langle f_{\mu \nu}^{1} \psi^{\mu} \psi^{\nu} e^{i p_{1} \cdot X}\left(z_{1}\right) a_{2} \cdot \partial X e^{i p_{2} \cdot X}\left(z_{2}\right) a_{3} \cdot \partial X e^{i p_{3} \cdot X}\left(z_{3}\right) a_{4} \cdot \partial X e^{i p_{4} \cdot X}\left(z_{4}\right)\right\rangle_{\text {even }}=0
$$

in any supersymmetric sector after summing over the even spin structures.

\subsection{Two fermion bilinears}

$$
\begin{aligned}
& \left\langle\frac{i}{2} f_{\mu_{1} \nu_{1}}^{1} \psi^{\mu_{1}} \psi^{\nu_{1}} e^{i p_{1} \cdot X}\left(z_{1}\right) \frac{i}{2} f_{\mu_{2} \nu_{2}}^{2} \psi^{\mu_{2}} \psi^{\nu_{2}} e^{i p_{2} \cdot X}\left(z_{2}\right) a_{3} \cdot \partial X e^{i p_{3} \cdot X}\left(z_{3}\right) a_{4} \cdot \partial X e^{i p_{4} \cdot X}\left(z_{4}\right)\right\rangle_{\text {even }} \\
& =-\frac{1}{2}\left(f_{1} f_{2}\right) \mathcal{E}_{\mathcal{N}} \Pi\left(z_{i} ; p_{i}\right)\left[a_{3} \cdot a_{4} \partial_{3} \partial_{4} \mathcal{G}_{34}-\sum_{i \neq 3} a_{3} \cdot p_{i} \partial_{3} \mathcal{G}_{3 i} \sum_{j \neq 4} a_{4} \cdot p_{j} \partial_{4} \mathcal{G}_{4 j}\right]
\end{aligned}
$$

plus permutations (6 in all), where

$$
\Pi\left(p_{i}\right)=\prod_{i<j} \exp \left(-p_{i} \cdot p_{j} \mathcal{G}_{i j}\right)
$$

and, depending on the number of supersymmetries $\mathcal{N}$,

$$
\mathcal{E}_{\mathcal{N}=4}=0 \quad, \quad \mathcal{E}_{\mathcal{N}=2}=(2 \pi)^{2} \mathcal{X}_{a b}^{\mathcal{N}=2} \quad, \quad \mathcal{E}_{\mathcal{N}=1}=2 \pi \mathcal{X}_{a b}^{\mathcal{N}}=1 \frac{\mathcal{H}^{\prime}(0)}{\mathcal{H}(0)}
$$

with $\mathcal{H}(z)=\prod_{I} \theta_{1}\left(z+u_{a b}^{I}\right)$ and, up to $\delta\left(\sum_{i} p_{i}\right)$,

$$
\mathcal{X}_{a b}^{\mathcal{N}=4}=\frac{(2 \pi)^{4} \Lambda_{a b}}{4 n\left(\alpha^{\prime} t\right)^{2}}, \quad \mathcal{X}_{a b}^{\mathcal{N}=2}=\frac{(2 \pi)^{4} \mathcal{I}_{a b}^{\perp} \Lambda_{a b}^{\|}}{4 n\left(\alpha^{\prime} t\right)^{2}}, \quad \mathcal{X}_{a b}^{\mathcal{N}=1}=\frac{(2 \pi)^{4} \mathcal{I}_{a b}}{4 n\left(\alpha^{\prime} t\right)^{2}}
$$




\subsection{Three fermion bilinears}

$$
\begin{aligned}
& \left\langle\frac{i}{2} f_{\mu_{1} \nu_{1}}^{1} \psi^{\mu_{1}} \psi^{\nu_{1}} e^{i p_{1} \cdot X}\left(z_{1}\right) . . \frac{i}{2} f_{\mu_{3} \nu_{3}}^{3} \psi^{\mu_{3}} \psi^{\nu_{3}} e^{i p_{3} \cdot X}\left(z_{3}\right) a_{4} \cdot \partial X e^{i p_{4} \cdot X}\left(z_{4}\right)\right\rangle_{\text {even }} \\
& =\left(f_{1} f_{2} f_{3}\right) \mathcal{E}_{\mathcal{N}} \omega\left(z_{1}, z_{2}, z_{3}\right) \sum_{j \neq 4} a_{4} \cdot p_{j} \partial_{4} \mathcal{G}_{4 j} \Pi\left(z_{i} ; p_{i}\right)
\end{aligned}
$$

plus permutations (4 in all) where

$\omega\left(z_{1}, z_{2}, z_{3}\right)=\partial_{1} \log \theta_{1}\left(z_{12}\right)+\partial_{2} \log \theta_{1}\left(z_{23}\right)+\partial_{3} \log \theta_{1}\left(z_{31}\right)=\partial_{1} \mathcal{G}_{12}+\partial_{2} \mathcal{G}_{23}+\partial_{3} \mathcal{G}_{31}$.

\subsection{Four fermion bilinears, connected}

$$
\begin{aligned}
& \left\langle\frac{i}{2} f_{\mu_{1} \nu_{1}}^{1} \psi^{\mu_{1}} \psi^{\nu_{1}} e^{i p_{1} \cdot X}\left(z_{1}\right) \ldots \frac{i}{2} f_{\mu_{4} \nu_{4}}^{4} \psi^{\mu_{4}} \psi^{\nu_{4}} e^{i p_{4} \cdot X}\left(z_{4}\right)\right\rangle_{\text {even }}^{\text {conn }}=\frac{1}{2}\left(f_{1} f_{2} f_{3} f_{4}\right) \times \\
& \Pi\left(z_{i} ; p_{i}\right)\left[\mathcal{E}_{\mathcal{N}}\left[\mathcal{P}\left(z_{13}\right)-\omega\left(z_{1}, z_{2}, z_{3}\right) \omega\left(z_{1}, z_{4}, z_{3}\right)\right]+\mathcal{J}_{\mathcal{N}}+(1,3 \leftrightarrow 2,4)\right]
\end{aligned}
$$

plus permutations (3 in all) where

$$
\mathcal{J}_{\mathcal{N}=4}=(2 \pi)^{4} \Lambda_{a b}^{\|}, \quad \mathcal{J}_{\mathcal{N}=2}=-\mathcal{E}_{\mathcal{N}=2} \mathcal{P}\left(u_{a b}\right), \quad \mathcal{J}_{\mathcal{N}=1}=\mathcal{E}_{\mathcal{N}=1}\left[3 \eta_{1}+\frac{1}{6} \frac{\mathcal{H}^{\prime \prime \prime}(0)}{\mathcal{H}^{\prime}(0)}\right]
$$

with $\eta_{1}=-2 \pi i \partial_{\tau} \log \eta$.

\subsection{Four fermion bilinears, disconnected}

$$
\begin{aligned}
& \left\langle\frac{i}{2} f_{\mu_{1} \nu_{1}}^{1} \psi^{\mu_{1}} \psi^{\nu_{1}} e^{i p_{1} \cdot X}\left(z_{1}\right) \ldots \frac{i}{2} f_{\mu_{4} \nu_{4}}^{4} \psi^{\mu_{4}} \psi^{\nu_{4}} e^{i p_{4} \cdot X}\left(z_{4}\right)\right\rangle_{\text {even }}^{\text {disc }}= \\
& \frac{1}{4}\left(f_{1} f_{2}\right)\left(f_{3} f_{4}\right) \Pi\left(z_{i} ; p_{i}\right)\left\{\mathcal{E}_{\mathcal{N}}\left[\mathcal{P}\left(z_{12}\right)+\mathcal{P}\left(z_{34}\right)\right]-\mathcal{J}_{\mathcal{N}}\right\}
\end{aligned}
$$

plus permutations (3 in all).

\section{CP odd amplitudes in $\mathcal{N}=1$ sectors}

In the odd spin structure, the presence of a supermodulus requires the insertion of $\delta(\beta)=e^{+\varphi}$ in order to absorb the zero mode of the anti-superghost $\beta=e^{-\varphi} \partial \xi$. The presence of a conformal Killing spinor requires the insertion of $\delta(\gamma)=e^{-\varphi}$ in order to absorb the zero mode of the superghost $\gamma=\eta e^{+\varphi}$. This allows one to fix the position in superspace of one of the vertices that would than be of the form $V=a \cdot \psi \exp (i p X)$. The two combined operations are equivalent to inserting a picture changing operator $\Gamma=e^{\varphi} G+\ldots$, where $G$ is the worldsheet supercurrent, at an arbitrary point $z_{0}$ and using the $(-1)$ picture for one of the vertices. Independence from $z_{0}$ allows one to let

$z_{0}$ coincide with the position of the vertex in the $(-1)$ picture and replace it with the 
expression in the (0) picture after using $\left\langle e^{\varphi(z)} e^{-\varphi(w)}\right\rangle_{\text {odd }}=1$. Moreover one has to absorb the four zero-modes of the spacetime fermions present in $\mathcal{N}=1$ sectors. In $\mathcal{N}=2$ and $\mathcal{N}=4$ sectors, $\mathrm{CP}$ odd amplitudes with only vector bosons vanish. There is no way to absorb the two (for $\mathcal{N}=2$ ) or six (for $\mathcal{N}=4$ ) additional zero-modes of the internal fermions present in these sectors. More complicated amplitudes with matter scalars and fermions can accomplish the task.

Let us thus concentrate on $\mathcal{N}=1$ sectors and start from the simplest non vanishing contribution.

\subsection{Two fermion bilinears ( 6 terms)}

Thanks to the exact cancellation between bosonic and fermionic non-zero modes on the worldsheet the final result for terms of the form $\langle\partial X \partial X: \psi \psi:: \psi \psi:\rangle_{\text {odd }}$ is very simple and compact

$$
\begin{aligned}
& \left\langle\partial X\left(z_{1}\right) \partial X\left(z_{2}\right): \psi \psi:\left(z_{3}\right): \psi \psi:\left(z_{4}\right)\right\rangle_{\text {odd }}=\frac{2}{2^{2}}\left(\sqrt{\frac{2}{\tau_{2}^{\mathcal{A}}}}\right)^{4}\left(f_{3} \cdot \tilde{f}_{4}\right) \mathcal{X}_{a b}^{\mathcal{N}=1} \times \\
& {\left[a_{1} \cdot a_{2} \partial_{1} \partial_{2} \mathcal{G}\left(z_{12}\right)-\sum_{i \neq 1} a_{1} \cdot p_{i} \partial_{1} \mathcal{G}\left(z_{1 i}\right) \sum_{j \neq 2} a_{2} \cdot p_{j} \partial_{2} \mathcal{G}\left(z_{2 j}\right)\right] \Pi\left(z_{i} ; p_{i}\right)}
\end{aligned}
$$

where $\Pi\left(z_{i} ; p_{i}\right)$ denotes the momentum factor (3.25) and the overall coefficient takes into account symmetry factors and the correct normalization of the fermionic zeromodes. In addition there are five more permutations.

\subsection{Three fermion bilinears (12 terms)}

The next simplest term is

$$
\left\langle: \psi \psi:\left(z_{1}\right): \psi \psi:\left(z_{2}\right): \psi \psi:\left(z_{3}\right) \partial X\left(z_{4}\right)\right\rangle_{o d d}
$$

The four zero-modes can be absorbed in three distinct ways. For instance, absorbing two of them at $z_{3}$, one at $z_{1}$ and one at $z_{2}$ and contracting the remaining two fermions at $z_{1}$ and $z_{2}$ yield

$$
\left\langle\partial X(\psi \psi)^{3}\right\rangle_{\text {odd }}=\frac{2^{3}}{2^{3}}\left(\sqrt{\frac{2}{\tau_{2}^{\mathcal{A}}}}\right)^{4}\left(f_{1} \cdot f_{2} \cdot \tilde{f}_{3}\right) \mathcal{X}_{a b}^{\mathcal{N}=1} \mathcal{S}\left(z_{12}\right) \Pi\left(p_{i} ; z_{i}\right) \sum_{i \neq 4} i a_{4} \cdot p_{i} \partial_{4} \mathcal{G}\left(z_{i 4}\right)
$$

plus two more permutations. In the odd spin structure the fermionic propagator can be taken to be 87, 88

$$
\mathcal{S}(z-w)=-\partial_{z} \mathcal{G}(z-w)=\partial_{z} \log \theta_{1}(z-w)+2 \pi i \frac{\operatorname{Im}(z-w)}{\operatorname{Im} \tau} .
$$




\subsection{Four fermion bilinears ( 21 terms, 3 structures)}

Finally the most laborious term is

$$
\left\langle: \psi \psi:\left(z_{1}\right): \psi \psi:\left(z_{2}\right): \psi \psi:\left(z_{3}\right): \psi \psi:\left(z_{4}\right)\right\rangle_{\text {odd }} .
$$

In this case there are three possible ways of absorbing zero-modes.

Absorbing two zero-modes at one point (say $z_{1}$ ) and two at another point (say $z_{2}$ ), for a total of 6 permutations, contributes expressions of the form

$$
\left\langle(\psi \psi)^{4}\right\rangle_{\text {odd }}^{\left(2_{0} 2_{0} 00\right)}=-\frac{2^{2}}{2^{4}}\left(\sqrt{\frac{2}{\tau_{2}^{\mathcal{A}}}}\right)^{4}\left(f_{1} \tilde{f}_{2}\right)\left(f_{3} \cdot f_{4}\right) \mathcal{X}_{a b}^{\mathcal{N}=1} \mathcal{S}^{2}\left(z_{34}\right) \Pi\left(p_{i} ; z_{i}\right)
$$

plus permutations.

Next, one can absorb two zero-modes at one point (say $z_{1}$ ), one at another point (say $z_{2}$ ), and one at a third point (say $z_{3}$ ) for a total of 12 permutations contributing expressions of the form

$$
\left\langle(\psi \psi)^{4}\right\rangle_{\text {odd }}^{\left(2_{0} 1_{0} 1_{0} 0\right)}=\frac{2^{4}}{2^{4}}\left(\sqrt{\frac{2}{\tau_{2}^{\mathcal{A}}}}\right)^{4}\left(f_{2} \cdot \tilde{f}_{1} \cdot f_{3} \cdot f_{4}\right) \mathcal{X}_{a b}^{\mathcal{N}=1} \mathcal{S}\left(z_{24}\right) \mathcal{S}\left(z_{34}\right) \Pi\left(p_{i} ; z_{i}\right)
$$

plus permutations.

Finally one can absorb one zero mode at each point which yields

$$
\left\langle(\psi \psi)^{4}\right\rangle_{\text {odd }}^{\left(1_{10} 1_{0} 1_{0} 1_{0}\right)}=\frac{2^{4}}{2^{4}}\left(\sqrt{\frac{2}{\tau_{2}^{\mathcal{A}}}}\right)^{4} \epsilon_{\mu_{1} \mu_{2} \mu_{3} \mu_{4}} f_{1}^{\mu_{1} \nu} f_{2}^{\mu_{1}} f_{3}^{\mu_{3} \rho} f_{4}^{\mu_{4}}{ }_{\rho} \mathcal{X}_{a b}^{\mathcal{N}=1} \mathcal{S}\left(z_{12}\right) \mathcal{S}\left(z_{34}\right) \Pi\left(p_{i} ; z_{i}\right),
$$

as well as two more permutations arising from different Wick contractions of the fermionic non-zero modes.

\section{Forward scattering}

The recipe for computing string amplitudes requires integrating over the insertion points and then over the modular parameter(s) of the relevant Riemann surface. The task is prohibitively complicated, if not impossible, in general. Yet for some very special amplitudes or kinematic regimes the situation drastically simplifies. This is the case for non-planar forward scattering that, as we will see, allows to extract interesting predictions for near future colliders.

For forward scattering $p_{1}=-p_{4}, p_{2}=-p_{3}$. As a result there is only one non-zero kinematical invariant

$$
p_{1} \cdot p_{2}=p_{3} \cdot p_{4}=-p_{1} \cdot p_{3}=p_{2} \cdot p_{4}=-s / 2=u / 2 \quad p_{1} \cdot p_{4}=p_{2} \cdot p_{3}=0=t / 2
$$


so that

$$
\Pi\left(p_{i}, z_{i}\right) \rightarrow \Pi\left(s, z_{i}\right)=\exp \left(s / 2\left(\mathcal{G}_{12}-\mathcal{G}_{13}+\mathcal{G}_{34}-\mathcal{G}_{24}\right)\right.
$$

Moreover, since $a_{1}=a_{4}$ and $a_{2}=a_{3}$, one easily finds

$$
\left(f_{1} f_{1}\right)=\left(f_{4} f_{4}\right)=-\left(f_{1} f_{4}\right)=0 \quad, \quad\left(f_{2} f_{2}\right)=\left(f_{3} f_{3}\right)=-\left(f_{2} f_{3}\right)=0
$$

and

$$
\begin{aligned}
& \left(f_{1} \tilde{f}_{1}\right)=\left(f_{4} \tilde{f}_{4}\right)=-\left(f_{1} \tilde{f}_{4}\right)=\left(f_{4} \tilde{f}_{1}\right)=0 \\
& \left(f_{2} \tilde{f}_{2}\right)=\left(f_{3} \tilde{f}_{3}\right)=-\left(f_{2} \tilde{f}_{3}\right)=\left(f_{3} \tilde{f}_{2}\right)=0,
\end{aligned}
$$

in addition all cubic contractions vanish

$$
\left(f_{i} f_{j} f_{k}\right)=0 \quad, \quad\left(f_{i} f_{j} \tilde{f}_{k}\right)=0
$$

since at least two of the $f$ 's are equal (opposite). As a result contractions involving three fermion bilinears give vanishing contribution to forward scattering both to $\mathrm{CP}$ even and CP odd processes.

Moreover for non-planar amplitudes the two stacks of branes should be of the same kind $a=b$ so that $T_{1}=T_{4}^{\dagger}$ and $T_{2}=T_{3}^{\dagger}$ and $\operatorname{tr}_{a}\left(T_{1} T_{2}\right)=\operatorname{tr}_{b}\left(T_{3} T_{4}\right)$.

Notice that integration over the four points is unrestricted in the non-planar case, since the Chan-Paton factor $\operatorname{tr}\left(T_{1} T_{2}\right) \operatorname{tr}\left(T_{3} T_{4}\right)$ is invariant under re-ordering of $z_{1}, z_{2}$ and of $z_{3}, z_{4}$. So even if a priori $0<z_{1}<z_{2}<1$ for a given Chan-Paton factor $\operatorname{tr}\left(T_{1} T_{2}\right) \operatorname{tr}\left(T_{3} T_{4}\right)$, the other ordering $0<z_{2}<z_{1}<1$ has the same ChanPaton factor since $\operatorname{tr}\left(T_{1} T_{2}\right)=\operatorname{tr}\left(T_{2} T_{1}\right)$. This extends immediately to twisted sectors whereby $\operatorname{tr}\left(T_{1} T_{2} W^{k}\right) \operatorname{tr}\left(T_{3} T_{4} W^{k}\right)$ is also invariant under reordering since $\left[W, T_{i}\right]=0$. Integrating by parts is thus possible and further simplifies the non-planar forward scattering amplitudes.

\subsection{CP even amplitudes}

For forward scattering, dropping total derivatives and Chan-Paton factors but including all relevant permutations, CP even amplitudes with two fermion bilinears read

$$
\begin{aligned}
& \left\langle\frac{i}{2} f_{\mu_{1} \nu_{1}}^{1} \psi^{\mu_{1}} \psi^{\nu_{1}} e^{i p_{1} \cdot X\left(z_{1}\right)} \frac{i}{2} f_{\mu_{2} \nu_{2}}^{2} \psi^{\mu_{2}} \psi^{\nu_{2}} e^{i p_{2} \cdot X\left(z_{2}\right)} a_{2} \cdot \partial_{3} X e^{-i p_{2} \cdot X\left(z_{3}\right)} a_{1} \cdot \partial_{4} X e^{-i p_{1} \cdot X\left(z_{4}\right)}\right\rangle_{\text {even }}^{F S} \\
& + \text { perms }=-\frac{\left(f_{1} f_{2}\right)^{2}}{2 \alpha^{\prime} s} \mathcal{E}_{\mathcal{N}}\left[\partial_{1} \partial_{2} \mathcal{G}_{12}+\partial_{3} \partial_{4} \mathcal{G}_{34}+\partial_{1} \partial_{3} \mathcal{G}_{13}+\partial_{2} \partial_{4} \mathcal{G}_{24}\right] \Pi\left(z_{i} ; s\right),
\end{aligned}
$$

where, for shortness, $\mathcal{G}_{i j}=\mathcal{G}\left(z_{i j}\right)$ and

$$
\left(f_{1} f_{2}\right)=2\left[\left(a_{1} \cdot p_{2}\right)\left(a_{2} \cdot p_{1}\right)-\left(a_{1} \cdot a_{2}\right)\left(p_{1} \cdot p_{2}\right)\right] .
$$


CP even amplitudes arising from connected (box-type) contractions of four fermion bilinears can be conveniently simplified using the identity 87, 88

$$
\mathcal{B}_{\alpha}\left(z_{1}, z_{2}, z_{3}, z_{4}\right)+\mathcal{B}_{\alpha}\left(z_{1}, z_{3}, z_{2}, z_{4}\right)+\mathcal{B}_{\alpha}\left(z_{1}, z_{3}, z_{4}, z_{2}\right)=\left.\frac{1}{2} \partial_{z}^{4} \log \theta_{\alpha}(z)\right|_{z=0}
$$

where

$$
\mathcal{B}_{\alpha}\left(z_{1}, z_{2}, z_{3}, z_{4}\right)=\mathcal{S}_{\alpha}\left(z_{12}\right) \mathcal{S}_{\alpha}\left(z_{23}\right) \mathcal{S}_{\alpha}\left(z_{34}\right) \mathcal{S}_{\alpha}\left(z_{14}\right)
$$

and observing that for forward scattering

$$
t_{1}=\left(f_{1} f_{2} f_{3} f_{4}\right)=\left(f_{1} f_{2} f_{2} f_{1}\right)=\left(f_{1} f_{3} f_{2} f_{4}\right)=a_{1}^{2} a_{2}^{2}\left(p_{1} \cdot p_{2}\right)^{2}
$$

and

$$
t_{2}=\left(f_{1} f_{3} f_{4} f_{2}\right)=\left(f_{1} f_{2} f_{1} f_{2}\right)=\frac{1}{2}\left(f_{1} f_{2}\right)^{2}=2\left[\left(a_{1} \cdot p_{2}\right)\left(a_{2} \cdot p_{1}\right)-\left(a_{1} \cdot a_{2}\right)\left(p_{1} \cdot p_{2}\right)\right]^{2} .
$$

One then has

$$
\begin{aligned}
& \left\{t_{1}\left[\mathcal{B}_{\alpha}\left(z_{1}, z_{2}, z_{3}, z_{4}\right)+\mathcal{B}_{\alpha}\left(z_{1}, z_{3}, z_{2}, z_{4}\right)\right]+t_{2} \mathcal{B}_{a}\left(z_{1}, z_{3}, z_{4}, z_{2}\right)\right\} \mathcal{Z}_{\alpha} \\
& =\left.\frac{1}{2} t_{1} \mathcal{Z}_{\alpha} \partial_{z}^{4} \log \theta_{\alpha}(z)\right|_{z=0}+\left(t_{2}-t_{1}\right) \mathcal{B}_{a}\left(z_{1}, z_{3}, z_{4}, z_{2}\right) \mathcal{Z}_{\alpha} .
\end{aligned}
$$

Summing over the spin structures eventually yields

$$
\begin{aligned}
& \left\langle\frac{i}{2} f_{\mu_{1} \nu_{1}}^{1} \psi^{\mu_{1}} \psi^{\nu_{1}} e^{i p_{1} \cdot X\left(z_{1}\right)} \frac{i}{2} f_{\mu_{2} \nu_{2}}^{2} \psi^{\mu_{2}} \psi^{\nu_{2}} e^{i p_{2} \cdot X\left(z_{2}\right)}\right. \\
& \left.\frac{i}{2} f_{\mu_{3} \nu_{3}}^{2} \psi^{\mu_{3}} \psi^{\nu_{3}} e^{-i p_{2} \cdot X\left(z_{3}\right)} \frac{i}{2} f_{\mu_{4} \nu_{4}}^{1} \psi^{\mu_{4}} \psi^{\nu_{4}} e^{-i p_{4} \cdot X\left(z_{4}\right)}\right\rangle_{\text {even }}^{F S, \text { conn }}= \\
& \Pi\left(z_{i} ; s\right)\left\{\left(t_{2}-t_{1}\right) \mathcal{E}_{\mathcal{N}}\left[\mathcal{P}\left(z_{14}\right)-\omega\left(z_{1}, z_{4}, z_{2}\right) \omega\left(z_{1}, z_{4}, z_{3}\right)\right]+\left(t_{2}+2 t_{1}\right) \mathcal{J}_{\mathcal{N}}\right\} .
\end{aligned}
$$

Recall that symmetry under $(14) \leftrightarrow(23)$ exchange is expected and would follow if (3.59) hold.

For forward scattering the disconnected CP even contractions of four fermion bilinears yield

$$
\begin{aligned}
& \left\langle\frac{i}{2} f_{\mu_{1} \nu_{1}}^{1} \psi^{\mu_{1}} \psi^{\nu_{1}} e^{i p_{1} \cdot X\left(z_{1}\right)} \frac{i}{2} f_{\mu_{2} \nu_{2}}^{2} \psi^{\mu_{2}} \psi^{\nu_{2}} e^{i p_{2} \cdot X\left(z_{2}\right)}\right. \\
& \left.\frac{i}{2} f_{\mu_{3} \nu_{3}}^{2} \psi^{\mu_{3}} \psi^{\nu_{3}} e^{-i p_{2} \cdot X\left(z_{3}\right)} \frac{i}{2} f_{\mu_{4} \nu_{4}}^{1} \psi^{\mu_{4}} \psi^{\nu_{4}} e^{-i p_{4} \cdot X\left(z_{4}\right)}\right\rangle_{\text {even }}^{F S, d i s c}= \\
& \frac{1}{2} \Pi\left(z_{i} ; s\right) t_{2}\left\{\mathcal{E}_{\mathcal{N}}\left[\mathcal{P}\left(z_{12}\right)+\mathcal{P}\left(z_{34}\right)+\mathcal{P}\left(z_{13}\right)+\mathcal{P}\left(z_{24}\right)\right]-2 \mathcal{J}_{\mathcal{N}}\right\} .
\end{aligned}
$$

\subsection{CP odd amplitudes}

For forward scattering CP odd contractions of two fermion bilinears, after integrating by parts and including all the four non-vanishing permutations, one finds (recall $\left.\alpha^{\prime}=1 / 2\right)$

$$
\begin{gathered}
\langle\partial X \partial X: \psi \psi:: \psi \psi:\rangle_{\text {odd }}^{F S}=-\frac{\left(f_{1} f_{2}\right)\left(f_{1} \tilde{f}_{2}\right)}{4 \alpha^{\prime} s}\left(\sqrt{\frac{2}{\tau_{2}^{\mathcal{A}}}}\right)^{4} \mathcal{X}_{a b}^{\mathcal{N}=1} \times \\
\Pi\left(z_{i} ; s\right)\left[\partial_{1} \partial_{2} \mathcal{G}\left(z_{12}\right)+\partial_{3} \partial_{4} \mathcal{G}\left(z_{34}\right)+\partial_{1} \partial_{3} \mathcal{G}\left(z_{13}\right)+\partial_{2} \partial_{4} \mathcal{G}\left(z_{24}\right)\right]
\end{gathered}
$$


For forward scattering $\mathrm{CP}$ odd contractions of four fermion bilinears can be simplified by means of the identities

$$
\epsilon_{\mu_{1} \mu_{2} \mu_{3} \mu_{4}} f_{1}^{\mu_{1} \nu} \eta_{\nu \lambda} f_{2}^{\mu_{1} \lambda} f_{1}^{\mu_{3} \rho} \eta_{\rho \sigma} f_{2}^{\mu_{4} \sigma}=-\frac{1}{2}\left(f_{1} \tilde{f}_{2}\right)\left(f_{1} f_{2}\right)
$$

and

$$
\left(f_{1} \tilde{f}_{2} f_{1} f_{2}\right)=\frac{1}{2}\left(f_{1} \tilde{f}_{2}\right)\left(f_{1} f_{2}\right) .
$$

Thus eventually one gets

$$
\langle: \psi \psi:: \psi \psi:: \psi \psi:: \psi \psi:\rangle_{\text {odd }}^{F S}=\frac{1}{4}\left(f_{1} \tilde{f}_{2}\right)\left(f_{1} f_{2}\right) \mathcal{X}_{a b}^{\mathcal{N}=1}\left(\mathcal{S}_{12}-\mathcal{S}_{34}-\mathcal{S}_{13}+\mathcal{S}_{24}\right)^{2}
$$

\section{Imaginary part and total cross section}

According to the optical theorem, the total cross section for the production of closed string states in the bulk, obtains from the imaginary part of the non-planar forward scattering amplitude

$$
\sigma_{t o t}(s)=\frac{1}{s} \operatorname{Im} \mathcal{A}_{F S}(s)
$$

It turns out to prove convenient to transform the non-planar amplitude to the transverse channel that exposes the 'tree-level' unoriented closed string exchange. It is remarkable but not unexpected that our final compact expressions for the amplitudes transform covariantly, thus providing a check of their validity, if needed. Indeed, performing an S-modular transformation from $\tau=i t / 2$ to $\tilde{\tau}=i \ell$ ) one finds

$$
\mathcal{F}_{\mathcal{N}}(\tau=-1 / \tilde{\tau})=-i \tilde{\tau} \mathcal{F}_{\mathcal{N}}(\tilde{\tau})
$$

for all the correlators we have computed in any sector of the theory. The overall power of $\tilde{\tau}$ then cancels against the measure of integration $d t / t=d \ell / \ell$. Under the required S-modular transformation, the combinations $u_{a b}^{I}=k v_{a b}^{I}+\varepsilon_{a b}^{I} \tau_{\mathcal{A}}$ transform into $\tilde{u}_{a b}^{I}=k v_{a b}^{I} \tilde{\tau}_{\mathcal{A}}-\varepsilon_{a b}^{I}$. What was a projection in the direct channel becomes a mass-shift in the transverse channel and vice versa. The boundary insertion points $z_{1}$ and $z_{2}$ gets re-located onto a unit segment along the real axis, while $z_{3}$ and $z_{4}$ gets re-located onto a unit segment parallel to the real axis and displaced from it by an amount $\tilde{\tau}_{2} / 2=\ell / 2$.

Thanks to the symmetry of the Chan-Paton factors, the integration is unrestricted and total derivatives can be dropped since there is no boundary contribution. Indeed, terms of the form

$$
\partial_{1}\left[\mathcal{G}\left(z_{12}\right)-\mathcal{G}\left(z_{12}\right)\right] \partial_{4}\left[\mathcal{G}\left(z_{42}\right)-\mathcal{G}\left(z_{43}\right)\right] \Pi\left(s, z_{i}\right)=(2 / s)^{2} \partial_{1} \partial_{4} \Pi\left(s, z_{i}\right)
$$

being total derivatives integrate to zero. The relative sign appear due to $p_{3}=-p_{2}$. Similarly for 2,3 since $p_{4}=-p_{1}$. 
Terms of the form

$$
\partial_{1}\left[\mathcal{G}\left(z_{12}\right)-\mathcal{G}\left(z_{13}\right)\right] \partial_{2}\left[\mathcal{G}\left(z_{21}\right)-\mathcal{G}\left(z_{24}\right)\right] \Pi\left(s, z_{i}\right)
$$

are more involved. One has

$$
\begin{aligned}
& \partial_{1}\left[\mathcal{G}\left(z_{12}\right)-\mathcal{G}\left(z_{13}\right)\right] \partial_{2}\left[\mathcal{G}\left(z_{21}\right)-\mathcal{G}\left(z_{24}\right)\right] \Pi\left(s, z_{i}\right)=(2 / s) \partial_{1}\left[\mathcal{G}\left(z_{12}\right)-\mathcal{G}\left(z_{13}\right)\right] \partial_{2} \Pi\left(s, z_{i}\right) \\
& =(2 / s) \partial_{2}\left\{\partial_{1}\left[\mathcal{G}\left(z_{12}\right)-\mathcal{G}\left(z_{13}\right)\right] \Pi\left(s, z_{i}\right)\right\}-(2 / s) \partial_{2} \partial_{1}\left[\mathcal{G}\left(z_{12}\right)-\mathcal{G}\left(z_{13}\right)\right] \Pi\left(s, z_{i}\right) .
\end{aligned}
$$

The first term is a total derivative and integrates to zero. The second term can be conveniently rewritten as

$$
\partial_{2} \partial_{1} \mathcal{G}\left(z_{12}\right) \Pi\left(s, z_{i}\right)=-\frac{\alpha^{\prime}}{2}\left[\mathcal{P}\left(z_{12}\right)+2 \eta_{1}+\frac{\pi}{2 \tau_{2}^{\mathcal{A}}}\right] \Pi\left(s, z_{i}\right) .
$$

The same applies to the pairs of points $(1,3),(4,3)$ and $(2,4)$.

Dropping all the tildes for simplicity, the final form of the worldsheet integrals one needs to compute for the $\mathrm{CP}$ even case is

$$
\begin{aligned}
& \mathcal{A}_{\mathcal{N}}^{F S}(s)=\int d \ell \mathcal{X}_{\mathcal{N}} \int \prod_{i} d z_{i} e^{\frac{s}{2}\left(\mathcal{G}_{12}-\mathcal{G}_{13}+\mathcal{G}_{34}-\mathcal{G}_{24}\right)}\left\{2 t_{1} \mathcal{J}_{\mathcal{N}}+\frac{t_{2}}{\alpha^{\prime} s} \mathcal{E}_{\mathcal{N}}\left(4 \eta_{1}+\frac{\pi}{\ell}\right)+\right. \\
& \left.+\left(t_{2}-t_{1}\right) \mathcal{E}_{\mathcal{N}}\left[\mathcal{P}_{14}-\left(\partial_{1} \mathcal{G}_{14}\right)^{2}\right]+\frac{t_{2}}{2} \frac{\alpha^{\prime} s+1}{\alpha^{\prime} s} \mathcal{E}_{\mathcal{N}}\left[\mathcal{P}_{12}+\mathcal{P}_{34}+\mathcal{P}_{13}+\mathcal{P}_{24}\right]\right\}
\end{aligned}
$$

For $\mathcal{N}=4$ sectors, $\mathcal{E}_{\mathcal{N}=4}=0$ and only the first term contributes and yields

$$
\mathcal{A}_{\mathcal{N}=4}^{F S}=K_{\mathcal{N}=4}\left(s ; a_{1}, a_{2}\right) \int_{0}^{\infty} d \ell \frac{\tilde{\Lambda}_{6-\hat{d}}(\ell)}{\ell \hat{d} / 2} \prod_{i} \int_{0}^{1} d x_{i} \Pi_{X}\left(\ell ; z_{i}=x_{i}+i \delta_{i}\right),
$$

where

$$
K_{\mathcal{N}=4}\left(s ; a_{1}, a_{2}\right)=\frac{2 a_{1}^{2} a_{2}^{2}\left(p_{1} \cdot p_{2}\right)^{2}(2 \pi)^{4} \hat{V}_{6-\hat{d}}}{2_{G S O} 2_{\Omega} 2_{P_{4}}^{2} 2_{\Lambda_{6-\hat{d}}}^{3} N_{\text {orb }}} \operatorname{tr}\left(T_{1} T_{2}\right)^{2},
$$

$0 \leq \hat{d} \leq 6$ is the number of 'large' internal dimensions in the $D 3$-brane description, i.e. 'small' in the T-dual D9-brane description, and $\delta_{1}=\delta_{2}=0$ while $\delta_{3}=\delta_{4}=\ell / 2$. Dependence on the insertion points is only through $\Pi_{X}\left(\ell ; z_{i}\right)$.

Exploiting the series expansions in $q=e^{-2 \pi \ell}$ collected in an appendix, one finds

$$
\begin{aligned}
& {\left[4 q^{1 / 4} \sin \left(\pi x_{12}\right) \sin \left(\pi x_{34}\right)\right]^{\alpha^{\prime} s} \Pi_{X}(q)=1-2 \alpha^{\prime} s q^{1 / 2}\left[\cos \left(2 \pi x_{13}\right)+\cos \left(2 \pi x_{24}\right)\right]} \\
& +2 \alpha^{\prime} s q\left\{1+\cos \left(2 \pi x_{12}\right)+\cos \left(2 \pi x_{34}\right)+\right. \\
& \left.\left(\alpha^{\prime} s-1\right)\left[\cos ^{2}\left(2 \pi x_{13}\right)+\cos ^{2}\left(2 \pi x_{24}\right)\right]+2 \alpha^{\prime} s \cos \left(2 \pi x_{13}\right) \cos \left(2 \pi x_{24}\right)\right\} \\
& +\frac{4}{3} \alpha^{\prime} s q^{3 / 2}\left\{\left[1+\cos \left(2 \pi x_{12}\right)+\cos \left(2 \pi x_{34}\right)+\left(\alpha^{\prime} s-1\right) \cos \left(2 \pi x_{13}\right) \cos \left(2 \pi x_{24}\right)\right] \times\right. \\
& \left.\times 3 \alpha^{\prime} s\left[\cos \left(2 \pi x_{13}\right)+\cos \left(2 \pi x_{24}\right)\right]+\left(\alpha^{\prime} s-1\right)\left(\alpha^{\prime} s-2\right)\left[\cos ^{3}\left(2 \pi x_{13}\right)+\cos ^{3}\left(2 \pi x_{24}\right)\right]\right\} \\
& +\frac{1}{3} \alpha^{\prime} s q^{2}\left\{2\left(\alpha^{\prime} s-1\right)\left(\alpha^{\prime} s-2\right)\left(\alpha^{\prime} s-3\right)\left[\cos ^{4}\left(2 \pi x_{13}\right)+\cos ^{4}\left(2 \pi x_{24}\right)\right]+\right.
\end{aligned}
$$




$$
\begin{aligned}
& +8 \alpha^{\prime} s \cos \left(2 \pi x_{13}\right) \cos \left(2 \pi x_{24}\right)\left[\left(\alpha^{\prime} s-1\right)\left(\alpha^{\prime} s-2\right)\left(\cos ^{2}\left(2 \pi x_{13}\right)+\cos ^{2}\left(2 \pi x_{24}\right)\right)+\right. \\
& \left.3 \alpha^{\prime} s\left(1+\cos \left(2 \pi x_{12}\right)+\cos \left(2 \pi x_{34}\right)\right)\right]+ \\
& +12 \alpha^{\prime} s\left(\alpha^{\prime} s-1\right)^{2} \cos ^{2}\left(2 \pi x_{13}\right) \cos ^{2}\left(2 \pi x_{24}\right)+ \\
& +12\left[\cos ^{2}\left(2 \pi x_{13}\right)+\cos ^{2}\left(2 \pi x_{24}\right)\right]\left(1+\alpha^{\prime} s\left(\alpha^{\prime} s-1\right)\left[1+\cos \left(2 \pi x_{12}\right)+\cos \left(2 \pi x_{34}\right)\right]\right)+ \\
& +3\left\{2\left(\alpha^{\prime} s+1\right)\left[\cos \left(2 \pi x_{12}\right)+\cos \left(2 \pi x_{34}\right)\right]^{2}-4 \cos \left(2 \pi x_{12}\right) \cos \left(2 \pi x_{34}\right)+\right. \\
& \left.+2\left(2 \alpha^{\prime} s+1\right)\left[\cos \left(2 \pi x_{12}\right)+\cos \left(2 \pi x_{34}\right)\right]+2 \alpha^{\prime} s-3\right\}+\ldots
\end{aligned}
$$

Truncating to lowest order $\left(\right.$ i.e. $\left.q^{0}\right)$, performing the trigonometric integrals over the insertion points by means of ${ }^{5}$

$$
\int_{0}^{1} d x(\sin \pi x)^{a}(\cos \pi x)^{2 n}=\frac{\Gamma\left(\frac{1+a}{2}\right) \Gamma\left(\frac{1}{2}+n\right)}{\pi \Gamma\left(1+n+\frac{a}{2}\right)}
$$

and extracting the imaginary part by means of

$$
\operatorname{Im}\left(\int_{0}^{\infty} d \ell \ell^{-\alpha} e^{-\beta \ell}\right)=\frac{\pi \beta^{\alpha-1}}{\Gamma(\alpha)}
$$

one gets

$$
\sigma_{0}(s)=K_{\mathcal{N}=4}\left(s ; a_{1}, a_{2}\right) \frac{\pi}{s \Gamma\left(\frac{\hat{d}}{2}\right)}\left(-\frac{\pi \alpha^{\prime} s}{2}\right)^{\frac{\hat{d}}{2}-1}\left[\frac{\Gamma\left(\frac{1-\alpha^{\prime} s}{2}\right)}{2^{\alpha^{\prime} s} \sqrt{\pi} \Gamma\left(1-\frac{\alpha^{\prime} s}{2}\right)}\right]^{2}
$$

for $0 \leq \alpha^{\prime} s<4$, in perfect agreement with the results of [71, mutatis mutandis.

Integration over $x_{13}$ (equivalently $x_{24}$ ) effectively kills all half odd integer powers of $q$ in the expansion of $\Pi_{X}$, given in an appendix. The next contribution in $\mathcal{N}=4$ sectors thus comes from terms of order $q^{1}$. Performing the trigonometric integrals over the insertion points by means of (7.11) and extracting the imaginary part yields

$$
\sigma_{1}(s)=K_{\mathcal{N}=4}\left(s ; a_{1}, a_{2}\right) \frac{\pi}{s \Gamma\left(\frac{\hat{d}}{2}\right)}\left(-\frac{\pi\left(\alpha^{\prime} s-4\right)}{2}\right)^{\frac{\hat{d}}{2}-1}\left[\frac{\Gamma\left(\frac{1-\alpha^{\prime} s}{2}\right)}{2^{\alpha^{\prime} s} \sqrt{\pi} \Gamma\left(1-\frac{\alpha^{\prime} s}{2}\right)}\right]^{2} 2 B_{1}\left(\alpha^{\prime} s\right)
$$

for $4 \leq \alpha^{\prime} s<8$, where the 'form factor' is given by

$$
B_{1}\left(\alpha^{\prime} s\right)=2\left(\alpha^{\prime} s\right)^{2} \frac{\left(\alpha^{\prime} s\right)^{2}-3 \alpha^{\prime} s+4}{\left(\alpha^{\prime} s-2\right)^{2}}=2\left(\alpha^{\prime} s\right)^{2} \frac{\left(\alpha^{\prime} s-\frac{3}{2}\right)^{2}+\frac{7}{4}}{\left(\alpha^{\prime} s-2\right)^{2}},
$$

${ }^{5}$ It is easy too see that $\int_{0}^{1} d x(\sin \pi x)^{a}(\cos \pi x)^{2 n+1}=0$ or more generally

$$
\int_{0}^{1} d x(\sin \pi x)^{a}(\cos \pi x)^{b}=\frac{1+e^{i \pi b}}{2 \pi} \frac{\Gamma\left(\frac{1+a}{2}\right) \Gamma\left(\frac{1+b}{2}\right)}{\Gamma\left(1+\frac{a+b}{2}\right)}
$$


in perfect agreement with the results of [71], mutatis mutandis.

For $\mathcal{N}=2$ sectors the last term $\mathcal{J}_{\mathcal{N}=2}$ gives similar results after replacing the overall kinematical factor with

$$
K_{\mathcal{N}=2}\left(s ; a_{1}, a_{2}\right)=\frac{\pi^{2}}{3} \frac{2 a_{1}^{2} a_{2}^{2}\left(p_{1} \cdot p_{2}\right)^{2}(2 \pi)^{2} \mathcal{I}_{a b}^{\perp} \hat{V}_{2-\hat{d}}}{2_{G S O} 2_{\Omega} 2_{P_{4}}^{2} 2_{\Lambda_{2-\hat{d}}} N_{\text {orb }}} \operatorname{tr}\left(W_{k} T_{1} T_{2}\right)^{2}
$$

where the first factor comes from the constant term $\left(q^{0}\right)$ in the expansion of $-\mathcal{P}(u)$, and taking into account that the number of 'large' internal dimensions satisfies $0 \leq$ $\hat{d} \leq 2$ in these sectors.

For $\mathcal{N}=1$ sectors the last term $\mathcal{J}_{\mathcal{N}=1}$ gives poles rather than a cut since $\hat{d}=0$ in these sectors. The massless poles corresponds to the dependence of the gauge couplings on the VEV's of the massless closed string scalars in the twisted sectors. Massive poles signal the possibility of producing (unstable) closed string 'resonances' with not necessarily integer masses (in $1 / \alpha^{\prime}$ units).

A potential negative mass pole might arise from $\mathcal{P}\left(z_{12}\right)$ and similar terms. By OPE considerations however it should be absent. Reassuringly one finds this kind of terms with coefficient proportional to $1+\left(1 / \alpha^{\prime} s\right)$ so that

$$
\left(1+\frac{1}{\alpha^{\prime} s}\right) \int_{0}^{1}(\sin \pi x)^{-\alpha^{\prime}-2} d x=\frac{\Gamma\left(\frac{1-\alpha^{\prime} s}{2}\right)}{2^{\alpha^{\prime} s} \sqrt{\pi} \Gamma\left(1-\frac{\alpha^{\prime} s}{2}\right)}
$$

has no 'tachyonic' pole. The next term in the $q$ expansion of $P\left(z_{12}\right)$ is a constant $\left(-\pi^{2} / 3\right)$ that yields integrals of the form $(7.13)$. i.e. the form factor of the 'zero' mass states. The term of order $q$ is proportional to $\sin ^{2}(2 \pi x)$. Integration then yields

$$
\int_{0}^{1}(\sin \pi x)^{-\alpha^{\prime}+2} d x=\frac{\alpha^{\prime} s-1}{\alpha^{\prime} s-2} \frac{\Gamma\left(\frac{1-\alpha^{\prime} s}{2}\right)}{2^{\alpha^{\prime} s} \sqrt{\pi} \Gamma\left(1-\frac{\alpha^{\prime} s}{2}\right)} .
$$

The same situation prevails for the terms in $P\left(z_{34}\right)$.

Finally one should consider the combinations $\mathcal{P}\left(z_{14}\right)-\left(\partial_{1} \mathcal{G}_{14}\right)^{2}$, plus the corresponding ones with $(1,4)$ replaced by $(2,3)$. Quite remarkably

$$
\mathcal{P}\left(z_{14}\right)-\left(\partial_{1} \mathcal{G}_{14}\right)^{2}=-4 \pi i \frac{\partial}{\partial \tau} \log \left(\frac{\theta_{4}\left(x_{14} \mid \tau\right)}{\eta(\tau)}\right)
$$

that admits the following expansion

$$
\begin{aligned}
& \mathcal{P}\left(z_{14}\right)-\left(\partial_{1} \mathcal{G}_{14}\right)^{2}=-\frac{\pi^{2}}{3}-8 \pi^{2} \sum_{n, d_{n} \mid n} \frac{1-(-)^{n / d_{n}}}{2} q^{n / 2} \frac{n}{d_{n}} \cos \left(2 \pi d_{n} x_{14}\right) \\
& =-\frac{\pi^{2}}{3}-8 \pi^{2}\left[q^{1 / 2} \cos \left(2 \pi x_{14}\right)+q \cos \left(4 \pi x_{14}\right)+\ldots\right]
\end{aligned}
$$

where $d_{n} \mid n$ denotes the divisors of $n$. Combining with $\Pi_{X}$ one finds that half odd integer powers of $q$ vanish after integration over $d x_{13}$ or, equivalently, $d x_{24}$. However 
new integer powers are generated by combinations of integer and half odd integer powers of $q \Pi_{X}$ and $\mathcal{P}\left(z_{14}\right)-\left(\partial_{1} \mathcal{G}_{14}\right)^{2}$. For instance at order $q$ one finds

$$
\begin{aligned}
& -\frac{2 \pi^{2} \alpha^{\prime} s}{3}\left\{1+\cos \left(2 \pi x_{12}\right)+\cos \left(2 \pi x_{34}\right)+\right. \\
& \left.\left(\alpha^{\prime} s-1\right)\left[\cos ^{2}\left(2 \pi x_{13}\right)+\cos ^{2}\left(2 \pi x_{24}\right)\right]+2 \alpha^{\prime} s \cos \left(2 \pi x_{13}\right) \cos \left(2 \pi x_{24}\right)\right\} \\
& -8 \pi^{2} \cos \left(4 \pi x_{14}\right)+16 \pi^{2} \alpha^{\prime} s \cos \left(2 \pi x_{14}\right)\left[\cos \left(2 \pi x_{13}\right)+\cos \left(2 \pi x_{24}\right)\right] .
\end{aligned}
$$

After integration over $d x_{13}$ or, equivalently, $d x_{24}$, the last term gives $8 \pi^{2} \alpha^{\prime} s\left[\cos \left(2 \pi x_{34}\right)+\right.$ $\cos \left(2 \pi x_{12}\right)$ ], that modifies $\sigma_{1}(s)$ when combined with the lowest order term in ${ }^{6}$

$$
\mathcal{E}_{\mathcal{N}=1} \approx 3 \pi+2 \pi \sum_{I} q^{k v_{a b}^{I}}+4 \pi \sum_{I} q^{1-k v_{a b}^{I}}+\ldots
$$

New thresholds with fractional mass appear due to the fractional powers in the expansion of $\mathcal{E}_{\mathcal{N}}$.

Terms in $\mathcal{P}\left(z_{13}\right)$ and $\mathcal{P}\left(z_{24}\right)$ can be discussed similarly.

\section{Comments}

In their present form, our results are not directly related to processes observable at LHC. Without some recoiling observable (open string) states it is impossible to detect the decay into closed strings in the bulk. Yet it should not be difficult to include some soft observable particle along the lines of [71]. For hadronic colliders, such as LHC, a much subtler issue is how to extract hadronic cross sections from the 'partonic' cross sections we have computed. One has to convolute our or similar results with the partonic distributions of the relevant hadrons, i.e. the proton. To the best of our knowledge these are not known in analytic form but significant effort [89] is presently devoted into this important step.

At a more formal level, our results, obtained for a specific yet interesting class of supersymmetric models with open and unoriented strings, display a remarkably simple structure. This is largely due to the already observed fact that open string gauge bosons belong to the 'identity' sector of the internal conformal field theory, describing the compactification from $D=10$. We thus see no major obstacle in extending them to the case of genuinely interacting internal $\mathcal{N}=2 \mathrm{SCFT}$ 's, such as Gepner models 90]. It is tantalizing to speculate that

$$
\mathcal{E}^{(s)}=-\sum_{\alpha} c_{\alpha} e_{\alpha-1} \mathcal{Z}_{\alpha}^{(s)}
$$

and

$$
\mathcal{J}^{(s)}=\sum_{\alpha} c_{\alpha} e_{\alpha-1}^{2} \mathcal{Z}_{\alpha}^{(s)}
$$

\footnotetext{
${ }^{6}$ For simplicity we assume $k v_{a b}^{I}<1$.
} 
should remain valid, once the relevant partition functions $\mathcal{Z}_{\alpha}^{(s)}$, with $s$ ranging over all the sectors of the open string spectrum, are extracted from the supersymmetric characters

$$
\chi_{S U S Y}^{(s)}=\sum_{\alpha} c_{\alpha} \mathcal{Z}_{\alpha}^{(s)}=\sum_{\alpha} c_{\alpha} \frac{\theta_{\alpha}(0)}{\eta^{3}} \mathcal{W}_{\alpha}^{(s)},
$$

where $\mathcal{W}_{\alpha}^{(s)}$ denotes the contribution of the sector $s$ of the internal $\mathcal{N}=2$ SCFT in a given spin structure $\alpha$ 91, 92].

We would like to conclude with a comment on the supersymmetry properties of our amplitudes. Some time ago [93, Berkovits and Vallilo have proposed manifestly supersymmetric one-loop amplitudes for massless closed string states based on the hybrid formalism [94]. Deducing similar amplitudes for massless open string states should be straightforward in the hybrid formalism. However due to the factorization of the spacetime and internal SCFT's in the hybrid formalism, in the absence of $\mathrm{RR}$ fluxes, it is not clear to us how to reproduce the simple yet non trivial internal structures, such as the functions $\mathcal{E}_{\mathcal{N}=1}$ and $\mathcal{J}_{\mathcal{N}=1}$ that we have found in the NSR formalism. Other manifestly supersymmetric formalisms [67, 95] may help clarifying this issue. We leave it to future work with eyes wide open to the possibilities of dealing with RR fluxes [96 and the associated non-trivial warping arising in flux compactifications 97 .

\section{Acknowledgements}

We would like to thank Ya. Stanev, E. Trevigne, A. Kumar, M. Serone, E. Kiritsis, I. Klebanov for useful discussions. During completion of this work M.B. was visiting the Galileo Galilei Institute of Arcetri (FI), INFN is acknowledged for hospitality and hopefully for support. M.B. would also like to thank the organizers (C. Angelatonj, E. Dudas, T. Gherghetta and A. Pomarol) and the participants to the workshop "Beyond the Standard Model", especially R. Blumenhagen, for creating a stimulating environment on the expectations for $\mathrm{LHC}^{7}$. This work was supported in part by INFN, by the MIUR-COFIN contract 2003-023852, by the EU contracts MRTN-CT2004-503369 and MRTN-CT-2004-512194, by the INTAS contract 03-516346 and by the NATO grant PST.CLG.978785.

\footnotetext{
${ }^{7}$ The most credited version being now: L'italia Ha vinto il Campionato del mondo.
} 


\section{A. Elliptic functions}

\section{A.1 Definitions}

Let $q=e^{2 \pi i \tau}$ the Jacobi $\theta$-functions are defined as gaussian sums

$$
\theta\left[\begin{array}{l}
\alpha \\
\beta
\end{array}\right](z \mid \tau)=\sum_{n} q^{\frac{1}{2}(n+\alpha)^{2}} e^{2 \pi i(z-\beta)(n-\alpha)}
$$

where $\alpha \beta \in \mathbf{R}$.

Equivalently, for particular values of characteristics, such as $\alpha, \beta=0,1 / 2$ they are given also in terms of infinite product as follows

$$
\begin{aligned}
& \theta\left[\begin{array}{c}
\frac{1}{2} \\
\frac{1}{2}
\end{array}\right](z \mid \tau)=\theta_{1}(z \mid \tau)=2 q^{\frac{1}{8}} \sin (\pi z) \prod_{m=1}^{\infty}\left(1-q^{m}\right)\left(1-e^{2 \pi i z} q^{m}\right)\left(1-e^{-2 \pi i z} q^{m}\right) \\
& \theta\left[\begin{array}{c}
\frac{1}{2} \\
0
\end{array}\right](z \mid \tau)=\theta_{2}(z \mid \tau)=2 q^{\frac{1}{8}} \cos (\pi z) \prod_{m=1}^{\infty}\left(1-q^{m}\right)\left(1+e^{2 \pi i z} q^{m}\right)\left(1+e^{-2 \pi i z} q^{m}\right) \\
& \theta\left[\begin{array}{l}
0 \\
0
\end{array}\right](z \mid \tau)=\theta_{3}(z \mid \tau)=\prod_{m=1}^{\infty}\left(1-q^{m}\right)\left(1+e^{2 \pi i z} q^{m-\frac{1}{2}}\right)\left(1+e^{-2 \pi i z} q^{m-\frac{1}{2}}\right) \\
& \theta\left[\begin{array}{c}
0 \\
\frac{1}{2}
\end{array}\right](z \mid \tau)=\theta_{4}(z \mid \tau)=\prod_{m=1}^{\infty}\left(1-q^{m}\right)\left(1-e^{2 \pi i z} q^{m-\frac{1}{2}}\right)\left(1-e^{-2 \pi i z} q^{m-\frac{1}{2}}\right) .
\end{aligned}
$$

Dedekind $\eta$ function is defined as

$$
\eta(\tau)=q^{\frac{1}{24}} \prod_{n=1}^{\infty}\left(1-q^{n}\right)
$$

and satisfies $\theta_{1}^{\prime}(0)=2 \pi \eta^{3}$.

Weierstrass $\mathcal{P}$ function

$$
\mathcal{P}(z)=-\partial_{z}^{2} \log \theta_{1}(z)-2 \eta_{1}
$$

where

$$
\eta_{1}=-2 \pi i \frac{\partial}{\partial \tau} \log \eta=-\frac{1}{6} \frac{\theta_{1}^{\prime \prime \prime}(0)}{\theta_{1}^{\prime}(0)},
$$

has a double pole at $z=0$ and is bi-periodic in $z$.

The free fermionic propagator in the even spin structures (Szego kernel)

$$
\mathcal{S}_{\alpha}(z)=\frac{\theta_{\alpha}(z 0)}{\theta_{1}(z)} \frac{\theta_{1}^{\prime}(0)}{\theta_{\alpha}^{\prime}(0)}
$$

has a simple pole at $z=0$ and satisfies

$$
\mathcal{S}_{\alpha}(z)^{2}=\mathcal{P}(z)-e_{\alpha-1}
$$


where

$$
e_{\alpha-1}=4 \pi i \frac{\partial}{\partial \tau} \log \frac{\theta_{\alpha}(0)}{\eta}
$$

are also related to $\mathcal{P}(z)$ evaluated at the semiperiods

$$
e_{1}=\mathcal{P}\left(\frac{1}{2}\right), \quad e_{2}=\mathcal{P}\left(\frac{1+\tau}{2}\right), \quad e_{3}=\mathcal{P}\left(\frac{\tau}{2}\right)
$$

In the odd spin structure

$$
\mathcal{S}_{1}(z)=-\partial_{z} \mathcal{G}(z)
$$

is biperiodic with a simple pole but not analytic.

The free bosonic propagator (biperiodic with logarithmic behaviour at $z=0$ ) on the (covering) torus is given by

$$
\mathcal{G}(z)=-\frac{\alpha^{\prime}}{2}\left[\log \left|\frac{\theta_{1}(z)}{\theta_{1}^{\prime}(0)}\right|^{2}-\frac{2 \pi}{\operatorname{Im} \tau} \operatorname{Im} z^{2}\right] .
$$

\section{A.2 Pseudo-periodicity and zeroes}

Under lattice shifts of their first argument $z$, theta functions transform according to

$$
\begin{aligned}
& \theta\left[\begin{array}{l}
\alpha \\
\beta
\end{array}\right](z+1 \mid \tau)=e^{2 \pi i \alpha} \theta\left[\begin{array}{l}
\alpha \\
\beta
\end{array}\right](z \mid \tau) \\
& \theta\left[\begin{array}{l}
\alpha \\
\beta
\end{array}\right](z+\tau \mid \tau)=e^{-2 \pi i(z+\beta)-i \pi \tau} \theta\left[\begin{array}{l}
\alpha \\
\beta
\end{array}\right](z \mid \tau) .
\end{aligned}
$$

The location of their zeroes is given by

$$
\theta\left[\begin{array}{l}
\alpha \\
\beta
\end{array}\right](z \mid \tau)=0 \quad \leftrightarrow \quad z_{n, m}=\left(\alpha-\frac{1}{2}+n\right) \tau+\left(\beta-\frac{1}{2}+m\right) .
$$

\section{A.3 Modular Transformations}

Under $\mathrm{T}$ and $\mathrm{S}$ modular trasformations of their arguments theta functions transform according to

$$
\begin{aligned}
\theta\left[\begin{array}{l}
\alpha \\
\beta
\end{array}\right](z \mid \tau+1) & =e^{-i \pi \alpha(\alpha-1)} \theta\left[\begin{array}{l}
\alpha \\
\beta+\alpha-\frac{1}{2}
\end{array}\right](z \mid \tau) \\
\eta(\tau+1) & =e^{\frac{i \pi}{12}} \eta(\tau) \\
\theta\left[\begin{array}{c}
\alpha \\
\beta
\end{array}\right]\left(\frac{z}{\tau} \mid-\frac{1}{\tau}\right) & =(-i \tau)^{\frac{1}{2}} e^{2 i \pi \alpha \beta+i \pi z^{2} / \tau} \theta\left[\begin{array}{l}
\beta \\
-\alpha
\end{array}\right](z \mid \tau) \\
\eta\left(-\frac{1}{\tau}\right) & =(-i \tau)^{\frac{1}{2}} \eta(\tau) .
\end{aligned}
$$

The modular transformation $\mathrm{P}$, that connects the direct and transverse channel of Möbius strip amplitudes, is more involved. It consists in a sequence of $\mathrm{T}$ and $\mathrm{S}$ transformations $\left(P=T S T^{2} S\right)$ on the modular parameter $\tau_{M}=\frac{1}{2}+\frac{i t}{2}$

$$
\begin{aligned}
\theta\left[\begin{array}{c}
\alpha \\
\beta
\end{array}\right]\left(\frac{z}{i t} \mid \frac{1}{2}+\frac{i}{2 t}\right) & =e^{-i \pi \alpha(\alpha-1)-2 \pi i(\alpha+\beta-1 / 2)^{2}+2 \pi z^{2} / t} \sqrt{-i t} \theta\left[\begin{array}{c}
\alpha+2 \beta-2 \\
1 / 2-\alpha-\beta
\end{array}\right]\left(z \mid \frac{1}{2}+\frac{i t}{2}\right) \\
\eta\left(\frac{1}{2}+\frac{i}{2 t}\right) & =e^{i \pi / 4} \sqrt{-i t} \eta\left(\frac{1}{2}+\frac{i t}{2}\right) .
\end{aligned}
$$




\section{A.4 Useful Identities}

Riemann identity for even spin structures reads

$$
\begin{aligned}
& \sum_{\alpha} c_{\alpha} \theta_{\alpha}\left(z_{1}\right) \theta_{\alpha}\left(z_{2}\right) \theta_{\alpha}\left(z_{3}\right) \theta_{\alpha}\left(z_{4}\right)= \\
& \theta_{1}\left(z_{1}^{\prime}\right) \theta_{1}\left(z_{2}^{\prime}\right) \theta_{1}\left(z_{3}^{\prime}\right) \theta_{1}\left(z_{4}^{\prime}\right)-\theta_{1}\left(z_{1}^{\prime \prime}\right) \theta_{1}\left(z_{2}^{\prime \prime}\right) \theta_{1}\left(z_{3}^{\prime \prime}\right) \theta_{1}\left(z_{4}^{\prime \prime}\right),
\end{aligned}
$$

where $z_{i}^{\prime}$ and $z_{i}^{\prime \prime}$ are related to $z_{i}$ through

$$
\begin{aligned}
z_{1}^{\prime} & =\frac{1}{2}\left(z_{1}+z_{2}+z_{3}+z_{4}\right) & z_{2}^{\prime} & =\frac{1}{2}\left(z_{1}+z_{2}-z_{3}-z_{4}\right) \\
z_{3}^{\prime} & =\frac{1}{2}\left(z_{1}-z_{2}+z_{3}-z_{4}\right) & z_{4}^{\prime} & =\frac{1}{2}\left(z_{1}-z_{2}-z_{3}+z_{4}\right)
\end{aligned}
$$

and

$$
\begin{aligned}
& z_{1}^{\prime \prime}=\frac{1}{2}\left(-z_{1}+z_{2}+z_{3}+z_{4}\right) \quad z_{2}^{\prime \prime}=\frac{1}{2}\left(z_{1}-z_{2}+z_{3}+z_{4}\right) \\
& z_{3}^{\prime \prime}=\frac{1}{2}\left(z_{1}+z_{2}-z_{3}+z_{4}\right) \quad z_{4}^{\prime \prime}=\frac{1}{2}\left(z_{1}+z_{2}+z_{3}-z_{4}\right) .
\end{aligned}
$$

\section{A.5 Series Expansions}

Series expansion in powers of $q$ yield

$$
\begin{aligned}
\partial_{z} \log \theta_{1}(z, q) & =\pi \cot (\pi z)+4 \pi \sum_{n} \frac{q^{n}}{1-q^{n}} \sin (2 \pi n z) \\
& =\pi \operatorname{coth}(\pi z)+4 \pi \sum_{n, d_{n} \mid n} q^{n} \sin \left(2 \pi d_{n} z\right)
\end{aligned}
$$

and

$$
\begin{aligned}
\partial_{z}^{2} \log \theta_{1}(z, q) & =-\frac{\pi^{2}}{\sin (\pi z)^{2}}+8 \pi^{2} \sum_{n} \frac{n q^{n}}{1-q^{n}} \cos (2 \pi n z) \\
& =-\frac{\pi^{2}}{\sin (\pi z)^{2}}+8 \pi^{2} \sum_{n, d_{n} \mid n} q^{n} d_{n} \cos \left(2 \pi d_{n} z\right)
\end{aligned}
$$

where, using $\partial_{\tau} q=2 \pi i q$,

$$
\eta_{1} \equiv-2 \pi i \partial_{\tau} \log \eta=\frac{\pi^{2}}{6}-4 \pi^{2} \sum_{n} \frac{n q^{n}}{1-q^{n}}=\frac{\pi^{2}}{6}-4 \pi^{2} \sum_{n, d_{n} \mid n} q^{n} d_{n}
$$

so that

$$
\mathcal{P}(z) \equiv-\partial_{z}^{2} \log \theta_{1}(z, q)-2 \eta_{1}=\frac{\pi^{2}}{\sin (\pi z)^{2}}-\frac{\pi^{2}}{3}+8 \pi^{2} \sum_{n, d_{n} \mid n} q^{n} d_{n}\left[1-\cos \left(2 \pi d_{n} z\right)\right]
$$


Moreover

$$
4 \pi i \partial_{\tau} \log \theta_{1}(z, q)=-\pi^{2}+8 \pi^{2} \sum_{n, d_{n} \mid n} q^{n} \frac{n}{d_{n}}\left[1+2 \cos \left(2 \pi d_{n} z\right)\right]
$$

and

$$
\begin{aligned}
\left(\frac{\theta_{1}^{\prime}(z, q)}{\theta_{1}(z, q)}\right)^{2} & =\partial_{z}^{2} \log \theta_{1}(z, q)-\frac{\theta_{1}^{\prime \prime}(z, q)}{\theta_{1}(z, q)}=\partial_{z}^{2} \log \theta_{1}(z, q)-4 \pi i \partial_{\tau} \log \theta_{1}(z, q) \\
& =-\pi^{2} \cot (\pi z)^{2}-8 \pi^{2} \sum_{n, d_{n} \mid n} q^{n}\left[\left(\frac{2 n}{d_{n}}-d_{n}\right) \cos \left(2 \pi d_{n} z\right)+\frac{n}{d_{n}}\right]
\end{aligned}
$$

For points on different boundaries in the transverse channel $\theta_{1}$ gets effectively replaced by $\theta_{4}$ for which

$$
\begin{aligned}
\partial_{z} \log \theta_{4}(z, q) & =4 \pi \sum_{n} \frac{q^{n / 2}}{1-q^{n}} \sin (2 \pi n z) \\
& =4 \pi \sum_{n, d_{n} \mid n} q^{n / 2} \frac{1-(-)^{n / d_{n}}}{2} \sin \left(2 \pi d_{n} z\right)
\end{aligned}
$$

and

$$
\begin{aligned}
\partial_{z}^{2} \log \theta_{4}(z, q) & =8 \pi^{2} \sum_{n} \frac{n q^{n / 2}}{1-q^{n}} \cos (2 \pi n z) \\
& =8 \pi^{2} \sum_{n, d_{n} \mid n} q^{n / 2} \frac{1-(-)^{n / d_{n}}}{2} d_{n} \cos \left(2 \pi d_{n} z\right)
\end{aligned}
$$

Moreover

$$
\begin{aligned}
4 \pi i \partial_{\tau} \log \theta_{4}(z, q) & =8 \pi^{2} \sum_{n}\left[\frac{n q}{1-q^{n}}+\frac{2 q^{n} \cos (2 \pi n z)}{\left(1-q^{2 n}\right)^{2}}\right] \\
& =8 \pi^{2} \sum_{n, d_{n} \mid n}\left[d_{n} q^{n}+\frac{1-(-)^{n / d_{n}}}{2} \frac{n}{d_{n}} q^{n / 2} \cos \left(2 \pi d_{n} z\right)\right]
\end{aligned}
$$

so that

$$
\begin{aligned}
\left(\frac{\theta_{4}^{\prime}(z, q)}{\theta_{4}(z, q)}\right)^{2} & =\partial_{z}^{2} \log \theta_{4}(z, q)-\frac{\theta_{4}^{\prime \prime}(z, q)}{\theta_{4}(z, q)}=\partial_{z}^{2} \log \theta_{4}(z, q)-4 \pi i \frac{\partial_{\tau} \theta_{4}(z, q)}{\theta_{4}(z, q)} \\
& =-8 \pi^{2} \sum_{n, d_{n} \mid n}\left[d_{n} q^{n}+\frac{1-(-)^{n / d_{n}}}{2}\left(\frac{n}{d_{n}}-d_{n}\right) q^{n / 2} \cos \left(2 \pi d_{n} z\right)\right]
\end{aligned}
$$




\section{References}

[1] E. Dudas, Class. Quant. Grav. 17, R41 (2000) [arXiv:hep-ph/0006190].

[2] C. Angelantonj and A. Sagnotti, Phys. Rept. 371, 1 (2002) [Erratum-ibid. 376, 339 (2003)] [arXiv:hep-th/0204089].

[3] A. M. Uranga, "Chiral four-dimensional string compactifications with intersecting Class. Quant. Grav. 20, S373 (2003) [arXiv:hep-th/0301032].

[4] E. Kiritsis, Fortsch. Phys. 52, 200 (2004) [Phys. Rept. 421, 105 (2005 ERRAT,429,121-122.2006)] [arXiv:hep-th/0310001].

[5] C. Kokorelis, arXiv:hep-th/0402087.

[6] R. Blumenhagen, M. Cvetic, P. Langacker and G. Shiu, Ann. Rev. Nucl. Part. Sci. 55, 71 (2005) [arXiv:hep-th/0502005].

[7] A. Sagnotti, arXiv:hep-th/0208020.

[8] M. Bianchi and A. Sagnotti, Phys. Lett. B 211, 407 (1988).

[9] M. Bianchi and A. Sagnotti, Phys. Lett. B 231, 389 (1989).

[10] G. Pradisi and A. Sagnotti, Phys. Lett. B 216, 59 (1989).

[11] M. Bianchi and A. Sagnotti, Phys. Lett. B 247, 517 (1990).

[12] M. Bianchi and A. Sagnotti, Nucl. Phys. B 361, 519 (1991).

[13] M. Bianchi, G. Pradisi and A. Sagnotti, Phys. Lett. B 273, 389 (1991).

[14] M. Bianchi, G. Pradisi and A. Sagnotti, Nucl. Phys. B 376, 365 (1992).

[15] M. Bianchi, Ph. D. Thesis, University of Rome "Tor Vergata", preprint ROM2F-92/13.

[16] M. Bianchi, Nucl. Phys. B 528, 73 (1998) [arXiv:hep-th/9711201].

[17] E. Witten, JHEP 9802, 006 (1998) [arXiv:hep-th/9712028].

[18] J. Polchinski, Phys. Rev. Lett. 75, 4724 (1995) [arXiv:hep-th/9510017].

[19] E. G. Gimon and J. Polchinski, Phys. Rev. D 54, 1667 (1996) [arXiv:hep-th/9601038].

[20] J. Dai, R. G. Leigh and J. Polchinski, Mod. Phys. Lett. A 4, 2073 (1989).

[21] R. G. Leigh, Mod. Phys. Lett. A 4, 2767 (1989).

[22] M. Berkooz and R. G. Leigh, Nucl. Phys. B 483, 187 (1997) [arXiv:hep-th/9605049]. 
[23] C. Angelantonj, M. Bianchi, G. Pradisi, A. Sagnotti and Y. S. Stanev, Phys. Lett. B 385, 96 (1996) [arXiv:hep-th/9606169].

[24] Z. Kakushadze and G. Shiu, Phys. Rev. D 56, 3686 (1997) [arXiv:hep-th/9705163].

[25] Z. Kakushadze and G. Shiu, Nucl. Phys. B 520, 75 (1998) [arXiv:hep-th/9706051].

[26] G. Aldazabal, A. Font, L. E. Ibanez and G. Violero, Nucl. Phys. B 536, 29 (1998) [arXiv:hep-th/9804026].

[27] M. Bianchi, J. F. Morales and G. Pradisi, "Discrete torsion in non-geometric orbifolds and their open-string Nucl. Phys. B 573, 314 (2000)

[arXiv:hep-th/9910228].

[28] M. Berkooz, M. R. Douglas and R. G. Leigh, Nucl. Phys. B 480, 265 (1996) [arXiv:hep-th/9606139].

[29] M. Cvetic, G. Shiu and A. M. Uranga, Nucl. Phys. B 615, 3 (2001) [arXiv:hep-th/0107166].

[30] M. Cvetic, I. Papadimitriou and G. Shiu, Nucl. Phys. B 659 (2003) 193 [Erratum-ibid. B 696 (2004) 298] [arXiv:hep-th/0212177].

[31] R. Blumenhagen, Fortsch. Phys. 53, 426 (2005) [arXiv:hep-th/0412025].

[32] C. Angelantonj, M. Cardella and N. Irges, Nucl. Phys. B 725, 115 (2005) [arXiv:hep-th/0503179].

[33] V. Balasubramanian and R. G. Leigh, Phys. Rev. D 55, 6415 (1997) [arXiv:hep-th/9611165].

[34] M. Larosa and G. Pradisi, Nucl. Phys. B 667, 261 (2003) [arXiv:hep-th/0305224].

[35] F. Marchesano and G. Shiu, Phys. Rev. D 71, 011701 (2005) [arXiv:hep-th/0408059].

[36] F. Marchesano and G. Shiu, JHEP 0411, 041 (2004) [arXiv:hep-th/0409132].

[37] E. Dudas and C. Timirgaziu, Nucl. Phys. B 716, 65 (2005) [arXiv:hep-th/0502085].

[38] I. Antoniadis, N. Arkani-Hamed, S. Dimopoulos and G. R. Dvali, Phys. Lett. B 436, 257 (1998) [arXiv:hep-ph/9804398].

[39] N. Arkani-Hamed, S. Dimopoulos and G. R. Dvali, "Phenomenology, astrophysics and cosmology of theories with sub-millimeter Phys. Rev. D 59, 086004 (1999) [arXiv:hep-ph/9807344].

[40] K. R. Dienes, E. Dudas and T. Gherghetta, Nucl. Phys. B 537, 47 (1999) [arXiv:hep-ph/9806292].

[41] C. Bachas, arXiv:hep-th/9503030. 
[42] M. Bianchi and Y. S. Stanev, Nucl. Phys. B 523, 193 (1998)

[arXiv:hep-th/9711069].

[43] I. Antoniadis, E. Dudas and A. Sagnotti, Nucl. Phys. B 544, 469 (1999) [arXiv:hep-th/9807011].

[44] I. Antoniadis, G. D'Appollonio, E. Dudas and A. Sagnotti, Nucl. Phys. B 553, 133 (1999) [arXiv:hep-th/9812118].

[45] C. Angelantonj, AIP Conf. Proc. 751, 3 (2005) [arXiv:hep-th/0411085].

[46] S. B. Giddings, S. Kachru and J. Polchinski, Phys. Rev. D 66, 106006 (2002) [arXiv:hep-th/0105097].

[47] S. Kachru, M. B. Schulz and S. Trivedi, JHEP 0310, 007 (2003) [arXiv:hep-th/0201028].

[48] R. Blumenhagen, D. Lust and T. R. Taylor, Nucl. Phys. B 663, 319 (2003) [arXiv:hep-th/0303016].

[49] J. F. G. Cascales and A. M. Uranga, JHEP 0305, 011 (2003) [arXiv:hep-th/0303024].

[50] I. Antoniadis and T. Maillard, Nucl. Phys. B 716, 3 (2005) [arXiv:hep-th/0412008].

[51] R. Blumenhagen, M. Cvetic, F. Marchesano and G. Shiu, JHEP 0503, 050 (2005) [arXiv:hep-th/0502095].

[52] M. Bianchi and E. Trevigne, JHEP 0508, 034 (2005) [arXiv:hep-th/0502147].

[53] J. P. Derendinger, C. Kounnas, P. M. Petropoulos and F. Zwirner, Fortsch. Phys. 53, 926 (2005) [arXiv:hep-th/0503229].

[54] I. Antoniadis, A. Kumar and T. Maillard, arXiv:hep-th/0505260.

[55] P. G. Camara, A. Font and L. E. Ibanez, JHEP 0509, 013 (2005) [arXiv:hep-th/0506066].

[56] I. R. Klebanov and E. Witten, Nucl. Phys. B 664, 3 (2003) [arXiv:hep-th/0304079].

[57] D. Cremades, L. E. Ibanez and F. Marchesano, JHEP 0307, 038 (2003) [arXiv:hep-th/0302105].

[58] M. Cvetic and I. Papadimitriou, Phys. Rev. D 68 (2003) 046001 [Erratum-ibid. D 70 (2004) 029903] [arXiv:hep-th/0303083].

[59] D. Cremades, L. E. Ibanez and F. Marchesano, JHEP 0405, 079 (2004) [arXiv:hep-th/0404229].

[60] D. Lust, P. Mayr, R. Richter and S. Stieberger, Nucl. Phys. B 696, 205 (2004) [arXiv:hep-th/0404134]. 
[61] M. Bertolini, M. Billo, A. Lerda, J. F. Morales and R. Russo, Nucl. Phys. B 743, 1 (2006) [arXiv:hep-th/0512067].

[62] C. Bachas and C. Fabre, Nucl. Phys. B 476, 418 (1996) [arXiv:hep-th/9605028].

[63] I. Antoniadis, C. Bachas and E. Dudas, Nucl. Phys. B 560, 93 (1999) [arXiv:hep-th/9906039].

[64] D. Lust and S. Stieberger, arXiv:hep-th/0302221.

[65] S. A. Abel and B. W. Schofield, JHEP 0506, 072 (2005) [arXiv:hep-th/0412206].

[66] M. Bianchi and E. Trevigne, JHEP 0601, 092 (2006) [arXiv:hep-th/0506080].

[67] N. Berkovits and C. R. Mafra, "Some Superstring Amplitude Computations with the Non-Minimal Pure Spinor arXiv:hep-th/0607187.

[68] E. Dudas and J. Mourad, Nucl. Phys. B 575, 3 (2000) [arXiv:hep-th/9911019].

[69] S. Cullen, M. Perelstein and M. E. Peskin, Phys. Rev. D 62, 055012 (2000) [arXiv:hep-ph/0001166].

[70] E. Accomando, I. Antoniadis and K. Benakli, Nucl. Phys. B 579, 3 (2000) [arXiv:hep-ph/9912287].

[71] D. Chialva, R. Iengo and J. G. Russo, "Cross sections for production of closed superstrings at high energy Phys. Rev. D 71, 106009 (2005) [arXiv:hep-ph/0503125].

[72] D. Friedan, E. J. Martinec and S. H. Shenker, Nucl. Phys. B 271, 93 (1986).

[73] M. B. Green and J. H. Schwarz, Nucl. Phys. B 198, 441 (1982).

[74] J. H. Schwarz, Phys. Rept. 89, 223 (1982).

[75] M. Bianchi and A. V. Santini, to appear soon.

[76] T. Eguchi and H. Ooguri, Nucl. Phys. B 282, 308 (1987).

[77] A. Sagnotti, arXiv:hep-th/9302099.

[78] J. F. Morales, C. A. Scrucca and M. Serone, Nucl. Phys. B 552, 291 (1999) [arXiv:hep-th/9812071].

[79] C. A. Scrucca and M. Serone, Nucl. Phys. B 556, 197 (1999) [arXiv:hep-th/9903145].

[80] G. Aldazabal, D. Badagnani, L. E. Ibanez and A. M. Uranga, "Tadpole versus anomaly cancellation in $\mathrm{D}=4,6$ compact IIB JHEP 9906, 031 (1999) [arXiv:hep-th/9904071].

[81] M. Bianchi and J. F. Morales, JHEP 0003, 030 (2000) [arXiv:hep-th/0002149]. 
[82] L. E. Ibanez, R. Rabadan and A. M. Uranga, Nucl. Phys. B 542, 112 (1999) [arXiv:hep-th/9808139].

[83] I. Antoniadis, E. Kiritsis and J. Rizos, Nucl. Phys. B 637, 92 (2002) [arXiv:hep-th/0204153].

[84] P. Anastasopoulos, JHEP 0308, 005 (2003) [arXiv:hep-th/0306042].

[85] P. Anastasopoulos, M. Bianchi, E. Dudas and E. Kiritsis, arXiv:hep-th/0605225.

[86] A. Kumar, S. Mukhopadhyay and K. Ray, arXiv:hep-th/0605083.

[87] S. Stieberger and T. R. Taylor, Nucl. Phys. B 647 (2002) 49 [arXiv:hep-th/0207026];

[88] S. Stieberger and T. R. Taylor, "Non-Abelian Born-Infeld Action And Type I Heterotic Duality. Ii: Nucl. Phys. B 648 (2003) 3 [arXiv:hep-th/0209064].

[89] LHC Olympics at

[90] C. Angelantonj, M. Bianchi, G. Pradisi, A. Sagnotti and Y. S. Stanev, Phys. Lett. B 387, 743 (1996) [arXiv:hep-th/9607229].

[91] T. P. T. Dijkstra, L. R. Huiszoon and A. N. Schellekens, "Chiral supersymmetric standard model spectra from orientifolds of Gepner Phys. Lett. B 609, 408 (2005) [arXiv:hep-th/0403196].

[92] T. P. T. Dijkstra, L. R. Huiszoon and A. N. Schellekens, Nucl. Phys. B 710, 3 (2005) [arXiv:hep-th/0411129].

[93] N. Berkovits and B. C. Vallilo, "One loop N-point superstring amplitudes with manifest d $=4$ Nucl. Phys. B 624, 45 (2002) [arXiv:hep-th/0110168].

[94] N. Berkovits, arXiv:hep-th/9604123.

[95] C. R. Mafra, JHEP 0601 (2006) 075 [arXiv:hep-th/0512052].

[96] W. D. . Linch and B. C. Vallilo, arXiv:hep-th/0607122.

[97] M. Grana, Phys. Rept. 423, 91 (2006) [arXiv:hep-th/0509003]. 